\title{
The peculiar small-scale X-ray morphology of NGC 5846 observed with Chandra
}

\author{
G. Trinchieri ${ }^{1}$ and P. Goudfrooij ${ }^{2}$ \\ 1 Osservatorio Astronomico di Brera, via Brera 28, 20121 Milano, Italy \\ 2 Space Telescope Science Institute, 3700 San Martin Drive, Baltimore, MD 21218, USA
}

Received 15 January 2001 / Accepted 19 February 2002

\begin{abstract}
The excellent quality of the Chandra observation of NGC 5846 reveals a complex X-ray morphology of the central regions of this galaxy. An intriguing morphological similarity between the X-ray and the optical line emission, discovered before using ROSAT HRI images (Trinchieri et al. 1997), is confirmed here in unprecedented detail. Complex spectral characteristics are associated with the morphological peculiarities, indicating a possibly turbulent gas in this object. A population of $\sim 40$ individual sources is also observed, with $L_{x}$ in the range $\sim 3 \times 10^{38}-2 \times 10^{39} \mathrm{erg} \mathrm{s}^{-1}$, with an X-ray luminosity function that is steeper in the high-luminosity end than in other early-type galaxies.
\end{abstract}

Key words. galaxies: individual: NGC 5846 - X-rays: galaxies

\section{Introduction}

The hot interstellar medium (ISM) in bright early-type galaxies was first imaged with data from the Einstein Observatory, and a few of its broad characteristics have been established in the more recent past, mainly with observations from ROSAT and ASCA. Thermal emission from early type galaxies with a large $L_{x} / L_{B}$ ratio is characterized by an optically thin plasma spectrum, with typical average temperatures of $\sim 0.5-1 \mathrm{keV}$ (Forman et al. 1985; Canizares et al. 1987) and extent that could vary from galactic scale out to several hundred kpc. The hot ISM is not isothermal over the entire source, but can show a slow temperature increase over a large region (e.g., $\sim 40 \mathrm{kpc}$ in NGC 4636, Trinchieri et al. 1994), and its large-scale morphology can either be relatively smooth and regular, or show asymmetries and inhomogeneities (e.g., the characteristic "tail" in M86, Forman et al. 1979; also less dramatic distortions are found in other galaxies, see Trinchieri et al. 1994, 1997b; Kim \& Fabbiano 1995; and references therein). Several studies have focused on better understanding the hot gas properties in more detail, from the relation with the optical properties, galaxy density and grouping, to the chemical gas composition, all contributing to a vivacious and lively, though often controvertial, debate. On the other hand, the small-scale morphology and detailed characteristics of the ISM have so far been very poorly studied due to the limited spatial

Send offprint requests to: G. Trinchieri, e-mail: ginevra@brera.mi.astro.it resolution of the data available prior to the launch of the Chandra satellite and its instruments.

Coexisting with the hot ISM, a cooler ("warm") component has been mapped in $\mathrm{H} \alpha$ in several X-ray bright early-type galaxies (Kim 1989; Trinchieri \& Di Serego 1991; Shields 1991; Buson et al. 1993; Goudfrooij et al. 1994; Singh et al. 1995; Macchetto et al. 1996). The morphology of this emission can be either relatively smooth and azimuthally symmetric or exhibit filamentary structures, arcs and/or rings. Although clearly extended, it is generally confined to the innermost regions of the galaxies, often within $\sim 1 \mathrm{kpc}$, and it is relatively faint and hard to detect properly.

To date, the study of the relation between hotter and cooler gas has been restricted to a handful of galaxies. In particular, a very intriguing morphological similarity between X-ray and $\mathrm{H} \alpha$ emission has only been established in two normal galaxies not at the center of clusters, using ROSAT HRI observations (Trinchieri \& Noris 1995; Trinchieri et al. 1997a). These studies have shown that the highly asymmetric, clumpy and filamentary $\mathrm{H} \alpha$ emission in NGC 1553 and NGC 5846 correspond to a complex, structured, clumpy emission in the X-ray images, with gross similarities and some remarkably similar details as well. In NGC 4649 however, where the $\mathrm{H} \alpha$ emission is smooth and azimuthally symmetric, no structure or asymmetries are found in the X-ray emission.

Another intriguing suggestion from the X-ray data of early-type galaxies is the fact that these X-ray features may have different spectral characteristics. Global spectral variations with radius have been measured with the 
ROSAT PSPC, and indicate a variation of temperature, increasing towards larger radii. However, these variations are generally azimuthally averaged quantities derived on scales of arcminutes, and reliable information on small scales could typically not be derived from the X-ray instruments available up to now. An attempt at measuring a spectral variation in a small region was done using the PSPC observation of NGC 5846 (Trinchieri et al. 1997a): the gas in the region corresponding to a feature to the NE seemed to have a cooler temperature than the surrounding gas at the same galactocentric distance, indicating a cooler plasma in correspondence to enhanced emission.

The close similarity of the filamentary structures in $\mathrm{X}$-ray and optical emission in some early-type galaxies, together with the lack of them at both wavelengths in others, re-enforced the case for a physical connection between the different phases of the interstellar medium. Such a connection had been dismissed within the "cooling flow" model, since predicted $\mathrm{H} \alpha$ luminosities obtained from the cooling gas were significantly smaller than those actually observed (e.g., Baum 1992). Given the short cooling times of the X-ray-emitting gas, a plausible suggestion that has been made is that the hot gas is directly responsible for line emission through heat transfer from the hot gas to cooler gas through electron conduction (Sparks et al. 1989; de Jong et al. 1990). This scenario indeed explains in detail the observed enhancement of X-ray emission at the location of dusty optical filaments. In this respect, it is interesting to note that a filamentary dust lane in the central few kpc of NGC 5846 has been detected, with a morphology once again strikingly similar to that observed for the optical nebulosity and the X-ray emission (Goudfrooij \& Trinchieri 1998).

The high spatial and spectral resolution, sensitive data set that we have obtained using Chandra will be used in what follows to confirm the tentative results obtained with the ROSAT HRI and to better investigate the physical connection between different phases of the ISM in NGC 5846. This is a giant elliptical galaxy at the center of a small group of galaxies. Extended X-ray emission has already been detected from the galaxy and the group with the Einstein satellite (Biermann et al. 1989) and subsequently studied in detail with the ROSAT PSPC and ASCA (Finoguenov et al. 1999). We assume a distance of $31.6 \mathrm{Mpc}$, which gives a total $L_{\mathrm{x}}(0.2-2 \mathrm{keV})=$ $6 \times 10^{41} \mathrm{erg} \mathrm{s}^{-1}$ and a total gas mass $M_{\text {gas }} \sim 10^{11} M_{\odot}$ within 10' radius (from Finoguenov et al. 1999).

\section{Results of the Chandra data analysis}

We obtained an observation of NGC 5846 with the backilluminated S3 CCD (ACIS-S in imaging configuration) on board Chandra on May 24, 2000 for $\sim 30 \mathrm{ks}$. The data have been reprocessed using the new calibration files as
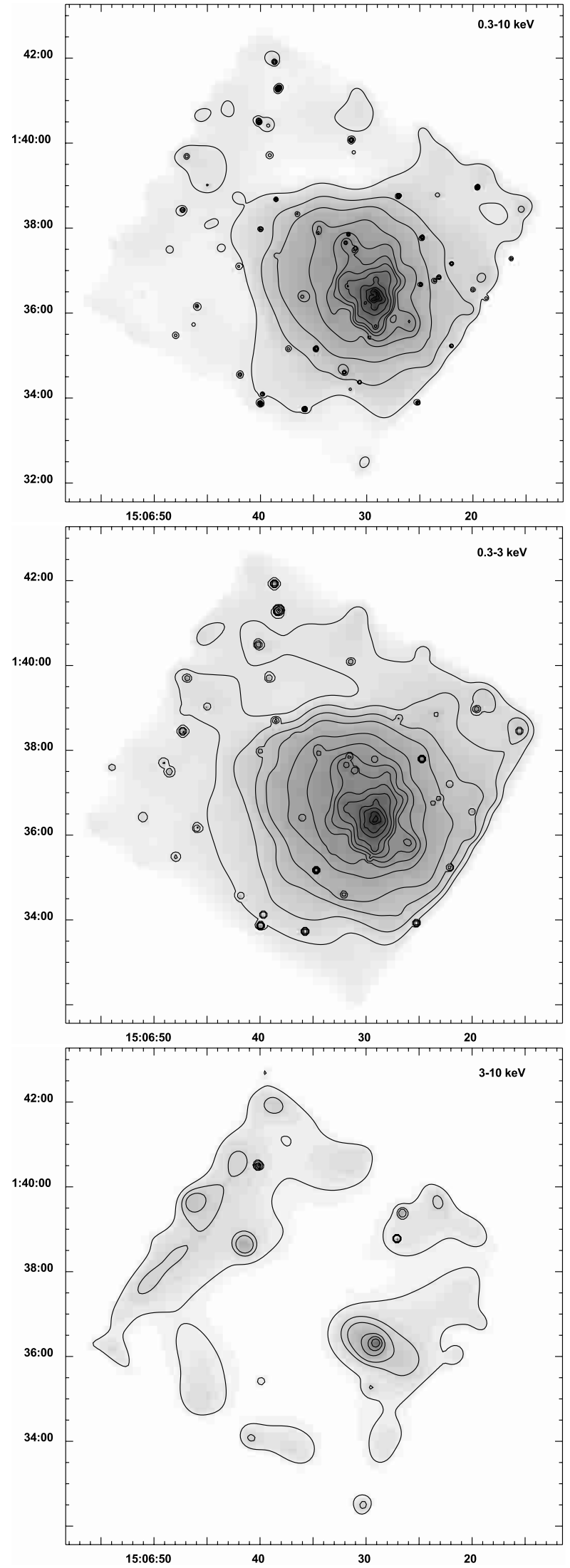

Fig. 1. Isointensity contour plot of the $\mathrm{X}$-ray emission in NGC 5846 in the full energy band $(0.3-10 \mathrm{keV})$, soft $(0.3-$ $3 \mathrm{keV})$ and hard $(3-10 \mathrm{keV})$. The data have been smoothed with an adaptive technique (fft method, with sigmin $=2.5$ and $\operatorname{sigmax}=5)$. 

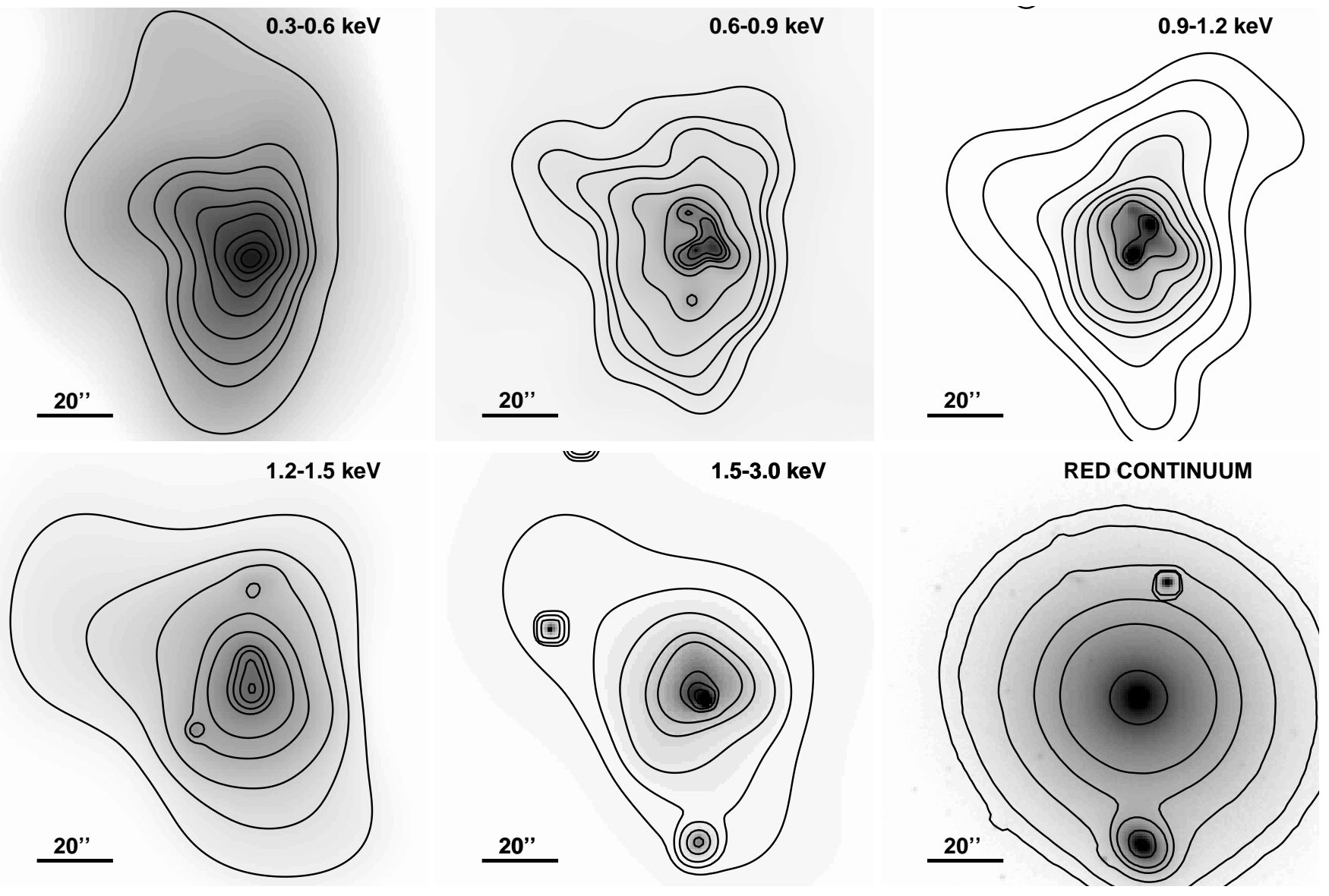

Fig. 2. Isointensity contour plots of the X-ray emission in the central region of NGC 5846. The different panels represent the data in energy bands within the $0.3-3 \mathrm{keV}$ range, binned to $1^{\prime \prime} \times 1^{\prime \prime}$ pixels and adaptively smoothed (fft kernel). The red optical continuum is shown in the bottom right panel on the same scale. Red continuum data were obtained with EFOSC1 at the ESO 3.6-m telescope (see Trinchieri \& di Serego Alighieri 1991).

explained in the CIAO documentation, and have been analyzed with both $\mathrm{CIAO}^{1}$ and the funtool/ds 9 software ${ }^{2}$.

We have followed the "CIAO Science Threads" given on the ASC home page to check and to improve the quality of the data obtained with the standard processing. In particular we have eliminated $\sim 5 \mathrm{ks}$ of data affected by a high background level, obtaining a net exposure time of $24264 \mathrm{~s}$.

Figure 1 presents the isocontours of the smoothed $\mathrm{X}$-ray image of the whole back-illuminated CCD in the full energy range $0.3-10 \mathrm{keV}$. The raw data have been smoothed with an adaptive filtering technique ("csmooth"). The X-ray emission from this object fills the entire CCD chip, and we expect it to extend outside, from previous X-ray images of the object in the soft energy band (cf. PSPC image, Trinchieri et al. 1997a; Finoguenov et al. 1999). However, since we are interested mostly on the detailed structure present in the X-ray morphology, and on a detailed study of the characteristics of the emission at small and medium scales, we will not extend the data analysis outside of the single back-illuminated S3 CCD.

\footnotetext{
1 http://asc.harvard.edu/ciao/

2 http://hea-www.harvard.edu/RD/index.html
}

\subsection{Maps}

Two broad energy band images $(0.3-3 \mathrm{keV}$ and $3-10 \mathrm{keV})$ are also shown in Fig. 1. It is quite clear from the comparison of the two maps that the emission is mostly confined below 2-3 keV, and that it has a general elongation to the NE and a rather complex morphology in the inner regions. Several small blobs can also be seen. These are most likely individual point sources, as will be discussed later (see Sect. 2.3). Several of these are also seen at higher energies, while the more diffuse component is almost entirely gone above $\sim 3 \mathrm{keV}$.

Close-ups of the inner region in different energy bands show more clearly the complexity of the morphology at small scales (Fig. 2). Strong deviations from azimuthal symmetry are evident in all energy bands, but the details of the pattern are not the same at all energies. The comparison with the central region of the galaxy in the optical is also quite striking, since none of the perturbations evident in the X-ray morphology is visible in the stellar light distribution.

The main feature of the X-ray emission is an arclike or "hook" structure at the very center which is composed of "blobs" of emission at slightly different 

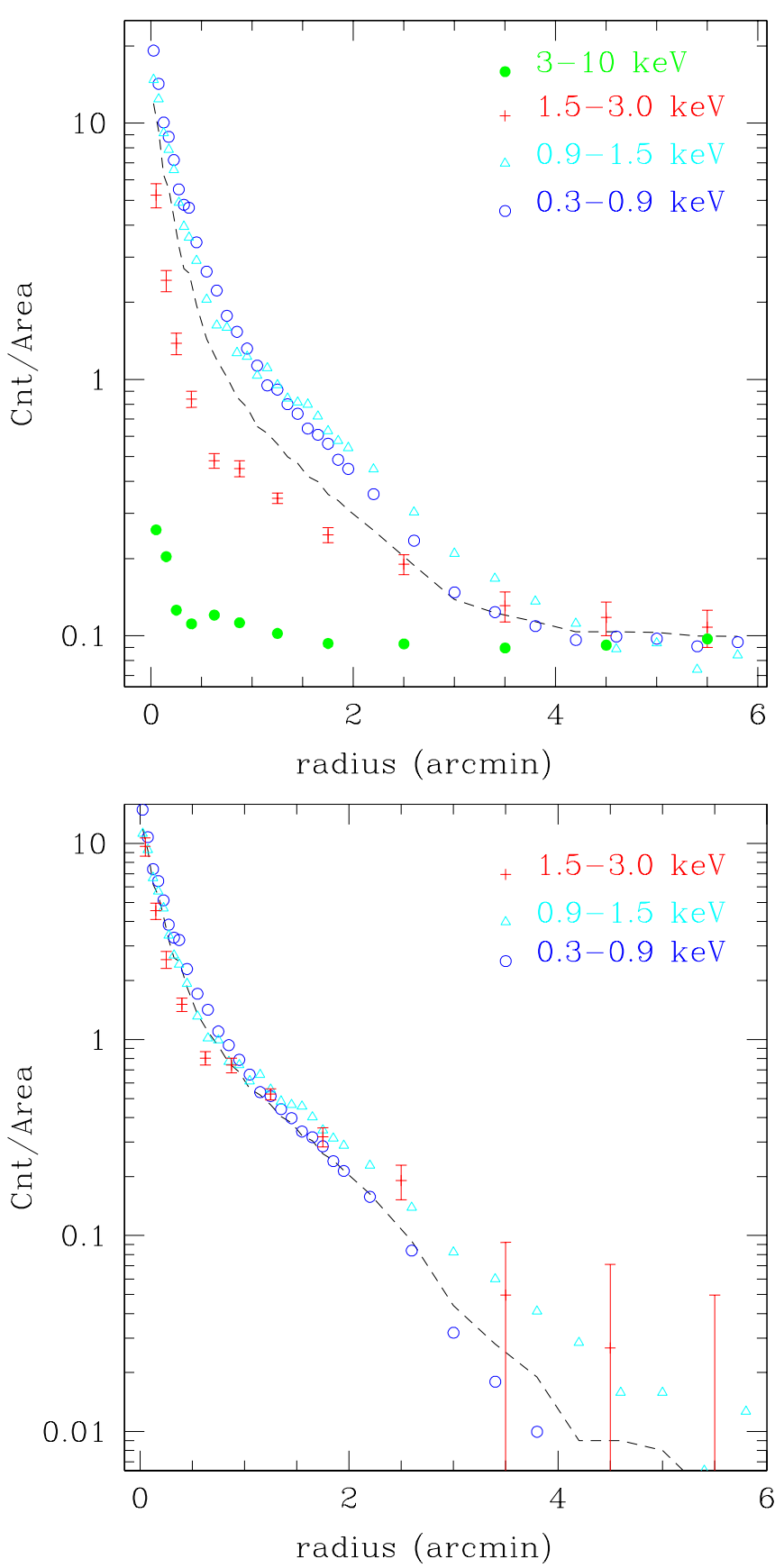

Fig. 3. Radial profile of the total (top) and net(bottom) emission in the energy bands indicated. The dashed line represents the profile in the full $0.3-10 \mathrm{keV}$ energy band. The profiles have been arbitrarily rescaled: to the background level in the top panel; to the peak value in the bottom one. Harder profiles are obtained in larger annuli than softer profiles, to retain better statistics. The net surface brightness in the hardest band $(>3 \mathrm{keV})$ is confined in the inner $1^{\prime}$ region and is not shown. Errors are indicated for the one band only (1.5-3 keV) only for clarity.

temperatures (or, more precise, with a different peak and shape in the photon distribution). A more coherent structure is visible at $>1^{\prime}$ distance from the central $\mathrm{X}$-ray peak, with an excess mostly to the NE, that again has no counterpart in the stellar light distribution. At hard Xrays $(k T \geq 3 \mathrm{keV})$, only the individual sources remain, and the coherent though distorted structure is no longer visible.

\subsection{Radial profiles}

We have derived azimuthally averaged radial profiles in several energy bands and in several azimuthal sectors, as shown in Figs. 3 and 4 . We centered the profiles at $(\mathrm{RA}, \mathrm{Dec})=\left(15^{\mathrm{h}} 06^{\mathrm{m}} 28.911,+01^{\mathrm{d}} 36^{\prime} 22^{\prime \prime} .35\right)$. The choice of the center is not straightforward, since there is no clearly defined peak common to all energies (see Fig. 2). However, since the central region is highly complex, we will disregard the innermost points for now. All point sources detected (see Sect. 2.3) have been excluded from the profile. Since the target is not located at the center of the $\mathrm{CCD}$, we have excluded regions not covered by the CCD by selecting photons within the largest rotated box consistent with the size and orientation of the chip. Therefore, the radial profile for radii larger than $\sim 2^{\prime}$ cover progressively smaller regions of the CCD.

In Fig. 3 we show the radial profiles of the raw counts, azimuthally averaged over $360^{\circ}$ and in 4 broad energy bands. Only at radii $r>4^{\prime}-5^{\prime}$ are the profiles constant with radius in all bands, indicating that emission is present over the almost entire field of view. At hard energies $(>3 \mathrm{keV})$, the profile appears to flatten at considerably smaller radii. To estimate the "field" background for this observation, consistently for all energies, we have chosen a small region in the $E$ corner, at a radius of $\gtrsim 5^{\prime}$ from the peak, where the surface brightness appears constant at all energy bands. In the total band, the value of the assumed background is $\sim 3.75 \times 10^{-6} \operatorname{cts~s}^{-1} \operatorname{arcsec}^{-2}$, higher than the expected ACIS-S background of $\sim 3 \times$ $10^{-6} \operatorname{cts~s}^{-1} \operatorname{arcsec}^{-2}$ (from blank sky fields). This could be due to background fluctuation in different sky regions, most likely with a contribution from the galaxy itself, that is known to extend well beyond the area covered by the S3 chip.

The radial distribution of the emission is rather complex, and appears to have different radial gradients, distribution and extent both as a function of energy (see Fig. 3) and as a function of angular sector (Fig. 4). In particular, the profile in the Eastern region is significantly higher, flatter and more complex than in the complementary Western sector, being most extended in the $40^{\circ}-80^{\circ}$ angular region. This is more pronounced at energies below $1.5 \mathrm{keV}$, and most in the $0.9-1.5 \mathrm{keV}$ band, which shows the greater extension and flatter overall distribution.

\subsection{Individual point sources}

The map in Fig. 1 indicates the presence of several individual point-like sources scattered over the full field of view, as listed in Table 1 . The source list was derived combining the results of different source detection algorithms 

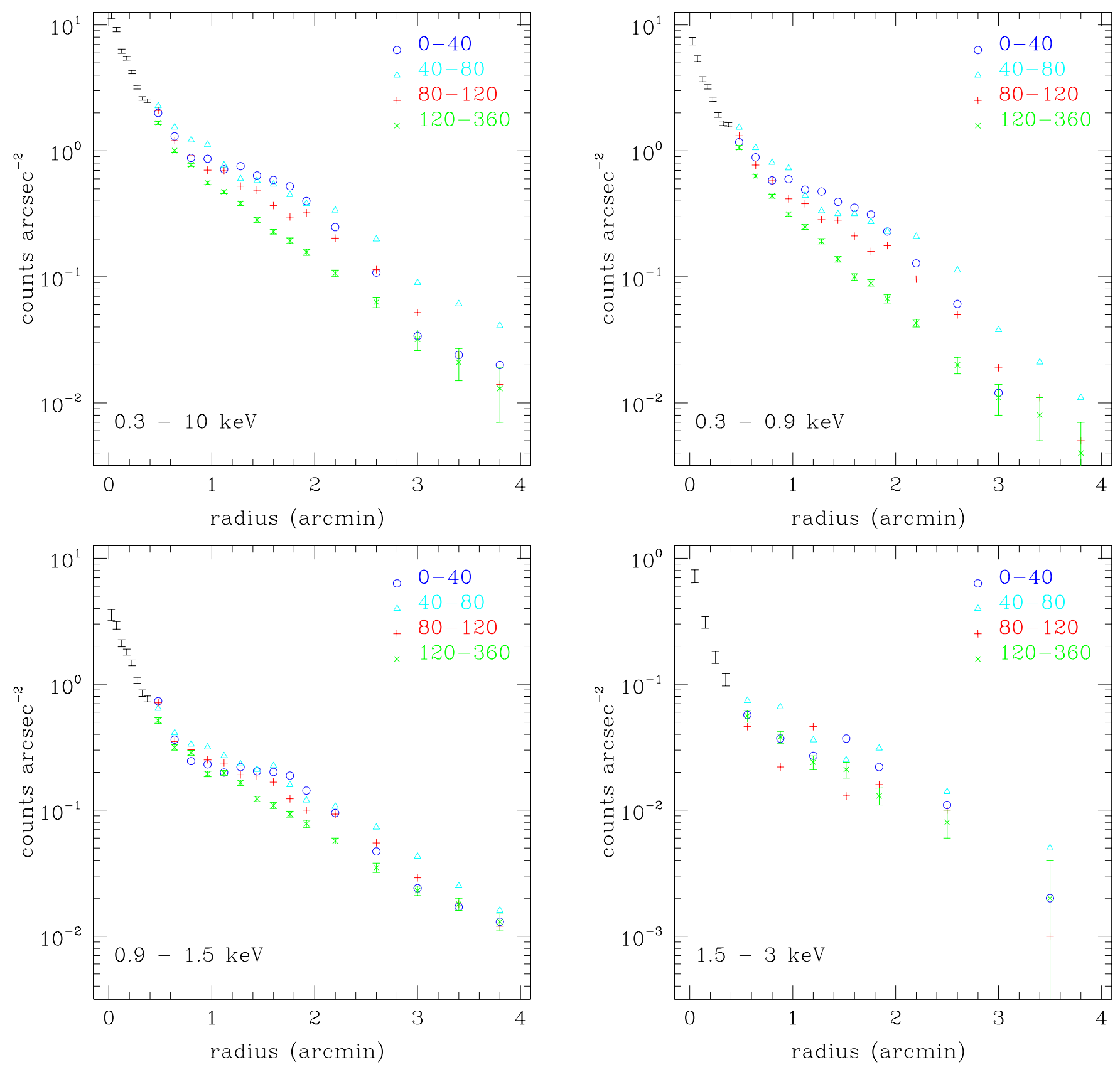

Fig. 4. Radial profile of the net emission in different azimuthal quadrants, as indicated. top left: $0.3-10 \mathrm{keV}$ band; top right: 0.3-0.9 keV; bottom Left: $0.9-1.5 \mathrm{keV}$; bottom right: $1.5-3.0 \mathrm{keV}$. The profiles are derived out to $r \sim 4^{\prime}$, but in the $120^{\circ}-360^{\circ}$ region the area covered by the CCD field of view is much smaller than in the Eastern profiles, due to the off-center position of the source.

provided by the CIAO software (celldetect, wavedetect) on the full energy band image, and on the two separate broad-band images used in Fig. 1. The combined list was cleaned of duplicates and compared with the adaptively smoothed images. A few sources that were evident from the map but were not included by any of the detection algorithms used were added. Net source counts were computed first in the full $0.3-10 \mathrm{keV}$ energy range, assuming as background the annular region at the same distance from the X-ray peak as the source, cleaned of the actual sources. The annuli chosen for this purpose are:
$0 ! 5-0.75,0.75-1^{\prime}, 1^{\prime}-2^{\prime}, 2^{\prime}-4^{\prime}$ and $>4^{\prime}$. Within 0.5 , both algorithms find many overlapping sources, grouped in a sort of elongated pattern in two main regions. We have substituted the many "individual" sources with two ellipses comprising the combined regions of the many individual sources, which we will consider later. In any case, the region within 0.5 is not considered at this stage. The list was further cleaned of sources with a signal-to-noise smaller than $\sim 3$. Source regions and their numbers (in increasing RA) are shown in Fig. 5, superposed onto the adaptively smoothed image. As can be seen from the plot, 


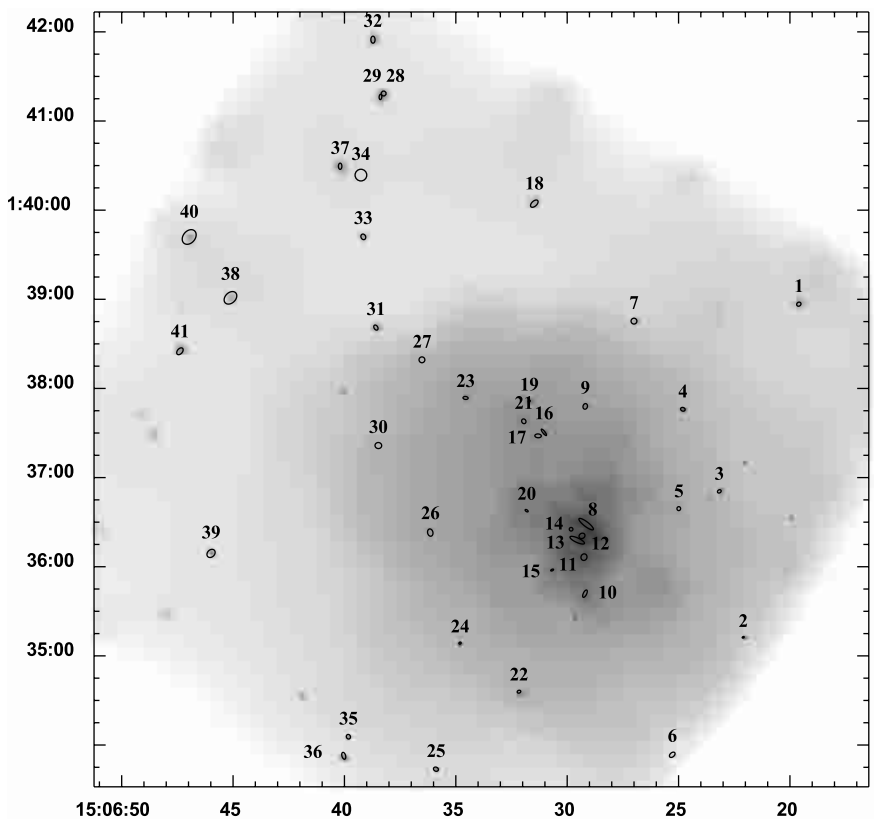

Fig. 5. The positions of the 41 sources are shown, together with the size and shape of the region used to determine their count rate. Sources are in order of increasing right ascension.

most peaks evident in the adaptively smoothed image are accounted for, while a few others are not included in the final list, because their signal-to-noise in the total band is smaller than our set threshold.

Since most of the detected sources have very few counts, attempts at determining fluxes or count rates in different energy bands have resulted in only a few detections. We therefore do not calculate hardness ratios for our detected sources. However, we notice that the distribution of the counts in broad energy bands could be very different in different sources (see also the discussion on hardness ratios in Blanton et al. 2001 and Sarazin et al. 2001).

Count rates in the total energy band are converted into fluxes assuming a constant conversion rate of 1 count $s^{-1}=7 \times 10^{-12} \mathrm{erg} \mathrm{cm}^{-2} \mathrm{~s}^{-1}$ (see Table 5). The $\mathrm{X}$-ray luminosities (Fig. 6) are distributed in the range $\sim 3 \times 10^{38} \lesssim L_{\mathrm{x}} \lesssim 2 \times 10^{39} \mathrm{erg} \mathrm{s}^{-1}$. Four sources in the inner $30^{\prime \prime}$ region (\# 8, 11, 12, 13) have not been included in the analysis of individual sources since they represent extended regions as discussed above. The total number of "individual" sources therefore is 37 . Although we do not claim completeness at the lower luminosities (see later), we should have detected all of the bright sources, and we have detected about 30 sources in the central $4^{\prime}$ radius of NGC 5846, which most likely all belong to the galaxy.

Figure 7 shows the luminosity function of the sources detected here. We have assumed that all are associated with NGC 5846, although we expect that a few of them might be (mostly background) intruders ( $<9$ for a flux limit $f_{0.5-2} \sim 2 \times 10^{-15} \mathrm{erg} \mathrm{s}^{-1}$ in the whole field of view, Hasinger et al. 1998). A power law distribution is suggested for luminosities above $\sim 4 \times 10^{38} \mathrm{erg} \mathrm{s}^{-1}$, with

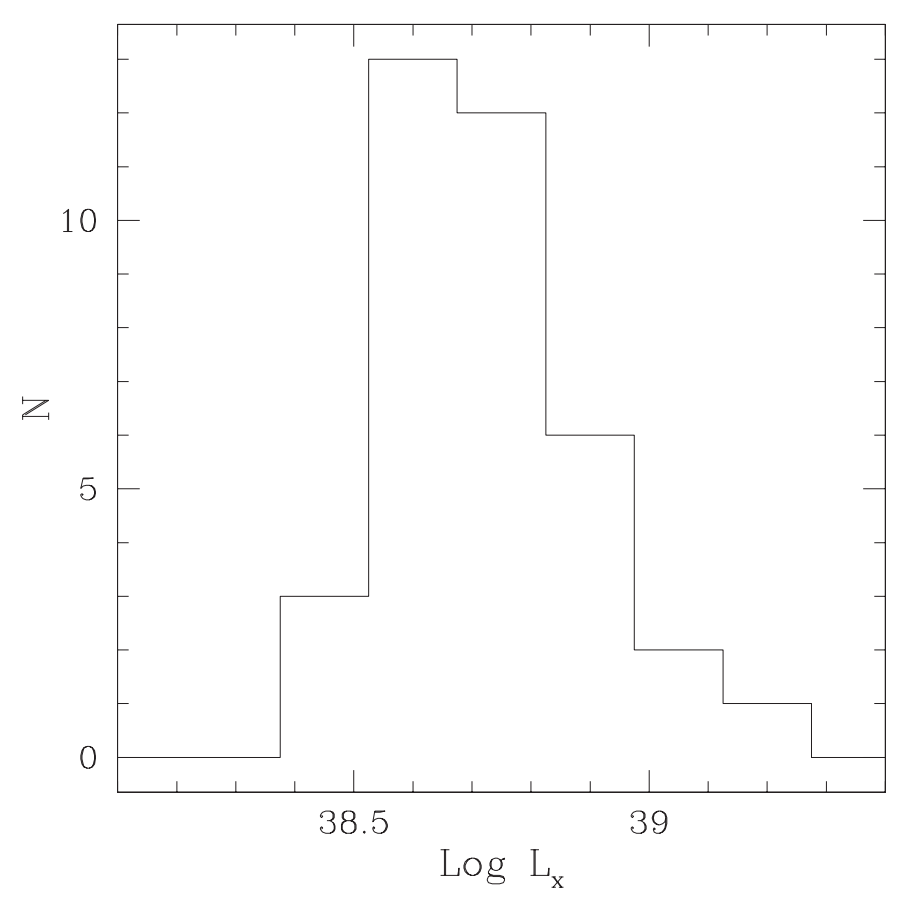

Fig. 6. The luminosity distribution of the sources detected in the total $(0.3-10 \mathrm{keV})$ energy band.

a turnover at lower luminosities. Although breaks in the luminosity functions of NGC 1553 and NGC 4697 have been suggested at similar luminosities (Blanton et al. 2001; Sarazin et al. 2000), in NGC 5846 sources at low luminosities (low count rates) are detected with less than or about 10 counts, and are already in most cases at the detection limit, so we cannot exclude a large bias due to incompleteness. The right panel in fact shows the luminosity function derived for sources detected at higher significance $(S / N>3.4)$. The flattening at low luminosity has disappeared almost completely. Also, the flattening becomes more conspicuous when sources of lower significance $(2.5 \sigma)$ are included (plot not shown).

The dashed line drawn over the steeper part of the luminosity function represents the slope $(-1.79)$ derived for the high-luminosity end of the luminosity function in M 31 (Shirey et al. 2001), which is the steepest value reported so far in the literature for early-type galaxies or bulges. In NGC 5846, an even steeper value of -2.4 might be more appropriate to cover the full range of luminosities sampled. Since the luminosity range sampled for NGC 1553 , NGC 4697, and NGC 5846 is very similar, this difference in slopes must indicate some other phenomenon at play.

\subsection{Energy distribution}

Given the complexity of the spectral characteristics of the extended emission, as already shown by the maps in Fig. 2, we defer a more complete treatment of the spectral data to a later paper. However, it should be noticed that the spectral characteristics of the gas are at least as complex as its morphological distribution. 
Table 1. Positions, net counts in the broad energy band $(0.3-10 \mathrm{keV})$, fluxes and luminosities $(0.3-10 \mathrm{keV})$ of the sources detected in NGC 5846. Sources inside of a radius $r=0.5$ from the center are large regions (ellipses) along and above structures evident in the X-ray maps (see Sect. 2.3 for details), and are within parenthesis in the table. Net counts are converted into fluxes assuming a conversion factor $2.6 \times 10^{-16} \mathrm{erg} \mathrm{cm}^{-2} \mathrm{~s}^{-1}$ counts $^{-1}$.

\begin{tabular}{|c|c|c|c|c|c|c|c|}
\hline $\begin{array}{l}\text { Source } \\
\text { Number }\end{array}$ & $\begin{array}{c}\text { RA } \\
(\mathrm{J} 2000)\end{array}$ & $\begin{array}{c}\text { Dec. } \\
(\mathrm{J} 2000)\end{array}$ & $\begin{array}{r}\text { Total } \\
\text { Counts }\end{array}$ & $\begin{array}{r}\text { Net } \\
\text { Counts }\end{array}$ & Error & $\begin{array}{c}f_{\mathrm{x}} \\
(0.3-10 \mathrm{keV})\end{array}$ & $L_{\mathrm{x}}$ \\
\hline 1 & $15: 06: 19.601$ & $+01: 38: 56.73$ & 15 & 14.1 & 3.8 & $3.7 \times 10^{15}$ & $4.5 \times 10^{38}$ \\
\hline 2 & $15: 06: 22.098$ & $+01: 35: 12.50$ & 10 & 9.8 & 3.2 & $2.6 \times 10^{15}$ & $3.1 \times 10^{38}$ \\
\hline 3 & $15: 06: 23.170$ & $+01: 36: 50.73$ & 14 & 12.1 & 3.7 & $3.1 \times 10^{15}$ & $3.9 \times 10^{38}$ \\
\hline 4 & $15: 06: 24.808$ & $+01: 37: 45.98$ & 54 & 51.4 & 7.3 & $1.34 \times 10^{14}$ & $1.64 \times 10^{39}$ \\
\hline 5 & $15: 06: 24.991$ & $+01: 36: 39.11$ & 15 & 12.4 & 3.8 & $3.2 \times 10^{15}$ & $3.9 \times 10^{38}$ \\
\hline 6 & $15: 06: 25.284$ & $+01: 33: 53.43$ & 15 & 13.5 & 3.8 & $3.5 \times 10^{15}$ & $4.3 \times 10^{38}$ \\
\hline 7 & $15: 06: 27.008$ & $+01: 38: 45.40$ & 24 & 22.1 & 4.9 & $5.8 \times 10^{15}$ & $7.1 \times 10^{38}$ \\
\hline$(8$ & $15: 06: 29.148$ & $+01: 36: 28.66$ & 393 & 291.9 & 19.9 & $7.59 \times 10^{14}$ & $\left.9.32 \times 10^{39}\right)$ \\
\hline 9 & $15: 06: 29.191$ & $+01: 37: 47.92$ & 17 & 13.0 & 4.1 & $3.4 \times 10^{15}$ & $4.2 \times 10^{38}$ \\
\hline 10 & $15: 06: 29.202$ & $+01: 35: 41.87$ & 47 & 35.1 & 6.9 & $9.1 \times 10^{15}$ & $1.12 \times 10^{39}$ \\
\hline (11 & $15: 06: 29.247$ & $+01: 36: 06.46$ & 101 & 55.3 & 10.1 & $1.44 \times 10^{14}$ & $\left.1.77 \times 10^{39}\right)$ \\
\hline$(12$ & $15: 06: 29.335$ & $+01: 36: 20.76$ & 173 & 136.9 & 13.2 & $3.56 \times 10^{14}$ & $4.37 \times 10^{39}$ \\
\hline$(13$ & $15: 06: 29.549$ & $+01: 36: 17.87$ & 304 & 221.4 & 17.5 & $5.76 \times 10^{14}$ & $\left.7.07 \times 10^{39}\right)$ \\
\hline 14 & $15: 06: 29.825$ & $+01: 36: 25.24$ & 40 & 24.8 & 6.3 & $6.4 \times 10^{15}$ & $7.9 \times 10^{38}$ \\
\hline 15 & $15: 06: 30.676$ & $+01: 35: 57.78$ & 15 & 13.2 & 3.9 & $3.4 \times 10^{15}$ & $4.2 \times 10^{38}$ \\
\hline 16 & $15: 06: 31.046$ & $+01: 37: 30.45$ & 41 & 38.2 & 6.4 & $9.9 \times 10^{15}$ & $1.22 \times 10^{39}$ \\
\hline 17 & $15: 06: 31.307$ & $+01: 37: 28.10$ & 24 & 20.2 & 4.9 & $5.3 \times 10^{15}$ & $6.5 \times 10^{38}$ \\
\hline 18 & $15: 06: 31.477$ & $+01: 40: 04.38$ & 21 & 18.5 & 4.6 & $4.8 \times 10^{15}$ & $5.9 \times 10^{38}$ \\
\hline 29 & $15: 06: 31.702$ & $+01: 37: 51.45$ & 15 & 14.6 & 3.9 & $3.8 \times 10^{15}$ & $4.7 \times 10^{38}$ \\
\hline 20 & $15: 06: 31.820$ & $+01: 36: 37.72$ & 30 & 27.0 & 5.5 & $7.0 \times 10^{15}$ & $8.6 \times 10^{38}$ \\
\hline 21 & $15: 06: 31.948$ & $+01: 37: 37.90$ & 22 & 18.9 & 4.7 & $4.9 \times 10^{15}$ & $6.1 \times 10^{38}$ \\
\hline 22 & $15: 06: 32.164$ & $+01: 34: 35.90$ & 20 & 18.5 & 4.5 & $4.8 \times 10^{15}$ & $5.9 \times 10^{38}$ \\
\hline 23 & $15: 06: 34.563$ & $+01: 37: 53.60$ & 18 & 15.7 & 4.2 & $4.1 \times 10^{15}$ & $5.0 \times 10^{38}$ \\
\hline 24 & $15: 06: 34.811$ & $+01: 35: 08.60$ & 25 & 24.5 & 5.0 & $6.4 \times 10^{15}$ & $7.8 \times 10^{38}$ \\
\hline 25 & $15: 06: 35.892$ & $+01: 33: 43.75$ & 25 & 23.8 & 5.0 & $6.2 \times 10^{15}$ & $7.6 \times 10^{38}$ \\
\hline 26 & $15: 06: 36.147$ & $+01: 36: 22.99$ & 21 & 14.9 & 4.6 & $3.9 \times 10^{15}$ & $4.7 \times 10^{38}$ \\
\hline 27 & $15: 06: 36.519$ & $+01: 38: 19.26$ & 15 & 13.1 & 3.9 & $3.4 \times 10^{15}$ & $4.2 \times 10^{38}$ \\
\hline 28 & $15: 06: 38.241$ & $+01: 41: 18.53$ & 13 & 12.2 & 3.6 & $3.2 \times 10^{15}$ & $3.9 \times 10^{38}$ \\
\hline 29 & $15: 06: 38.384$ & $+01: 41: 16.19$ & 16 & 15.6 & 4.0 & $4.0 \times 10^{15}$ & $5.0 \times 10^{38}$ \\
\hline 30 & $15: 06: 38.478$ & $+01: 37: 21.57$ & 13 & 10.8 & 3.6 & $2.8 \times 10^{15}$ & $3.4 \times 10^{38}$ \\
\hline 31 & $15: 06: 38.588$ & $+01: 38: 40.97$ & 16 & 14.8 & 4.0 & $3.9 \times 10^{15}$ & $4.7 \times 10^{38}$ \\
\hline 32 & $15: 06: 38.721$ & $+01: 41: 54.74$ & 16 & 14.9 & 4.0 & $3.9 \times 10^{15}$ & $4.8 \times 10^{38}$ \\
\hline 33 & $15: 06: 39.153$ & $+01: 39: 42.04$ & 12 & 11.0 & 3.5 & $2.9 \times 10^{15}$ & $3.5 \times 10^{38}$ \\
\hline 34 & $15: 06: 39.186$ & $+01: 40: 23.62$ & 22 & 17.1 & 4.7 & $4.4 \times 10^{15}$ & $5.5 \times 10^{38}$ \\
\hline 35 & $15: 06: 39.823$ & $+01: 34: 05.58$ & 11 & 10.0 & 3.3 & $2.6 \times 10^{15}$ & $3.2 \times 10^{38}$ \\
\hline 36 & $15: 06: 40.031$ & $+01: 33: 52.74$ & 23 & 21.5 & 4.8 & $5.6 \times 10^{15}$ & $6.9 \times 10^{38}$ \\
\hline 37 & $15: 06: 40.194$ & $+01: 40: 29.55$ & 20 & 19.2 & 4.5 & $5.0 \times 10^{15}$ & $6.1 \times 10^{38}$ \\
\hline 38 & $15: 06: 45.118$ & $+01: 39: 00.99$ & 18 & 12.5 & 4.2 & $3.3 \times 10^{15}$ & $4.0 \times 10^{38}$ \\
\hline 39 & $15: 06: 45.989$ & $+01: 36: 08.96$ & 16 & 13.7 & 4.0 & $3.6 \times 10^{15}$ & $4.4 \times 10^{38}$ \\
\hline 40 & $15: 06: 46.971$ & $+01: 39: 41.95$ & 25 & 17.9 & 5.0 & $4.7 \times 10^{15}$ & $5.7 \times 10^{38}$ \\
\hline 41 & $15: 06: 47.386$ & $+01: 38: 25.02$ & 11 & 9.6 & 3.3 & $2.5 \times 10^{15}$ & $3.1 \times 10^{38}$ \\
\hline
\end{tabular}

Figure 8 better illustrates the complexity of the emission on small scales. The energy distributions of the photons in four of the regions analyzed are compared. All regions selected are within a 0.75 radius from the center of the galaxy, and cover an area between $\sim 50 \operatorname{arcsec}^{2}$ and $\sim 300 \operatorname{arcsec}^{2}$. The data are binned in relatively large bins, to obtain a good statistical significance at each point and within the same energy boundaries, albeit at the expense of details ${ }^{3}$. The very center of the galaxy, covering the higher surface brightness structure and its adjacent lower surface brightness region. The other two are at slightly larger radial distance and cover the ENE and the $\mathrm{S}$ enhancements visible in the maps (Fig. 2). It is

\footnotetext{
3 The spectral matrixes for each region do not take into account the extended nature of the sources. While formally incorrect, the errors introduced by this assumption are very small on the scale of the sources analyzed here, and are also not very significant throughout the S3 chip (J. McDowell, private communication, see also Hicks et al. 2002).
}

evident from the figure that the distributions are not the same, both in shape and in the position of the peak. The average distribution at the same radial distance also appears to have a broader shape than for example the $\mathrm{S}$ region (open squares).

Even more dramatic is the comparison of the photon distribution on larger areas and at large radii. The two lower panels of Fig. 9 show the comparison between two inner regions, characterized by higher and lower surface brightness (the former being along the high surface brightness feature, inclusive of sources $8,12,13$; the latter in the lower surface brightness region to the ENE of it). The upper panel of Fig. 9 shows the energy distribution in three outer concentric annuli. These are defined as having inner/outer radii of $0.75-1^{\prime} ; 1^{\prime}-2^{\prime} ; 2^{\prime}-4^{\prime}$ respectively; all detected sources have been removed. The background is estimated from the easternmost corner of the CCD, at galactocentric radii of $>4^{\prime}$ and subtracted from the data; for all annuli care has been taken to ensure that the area 

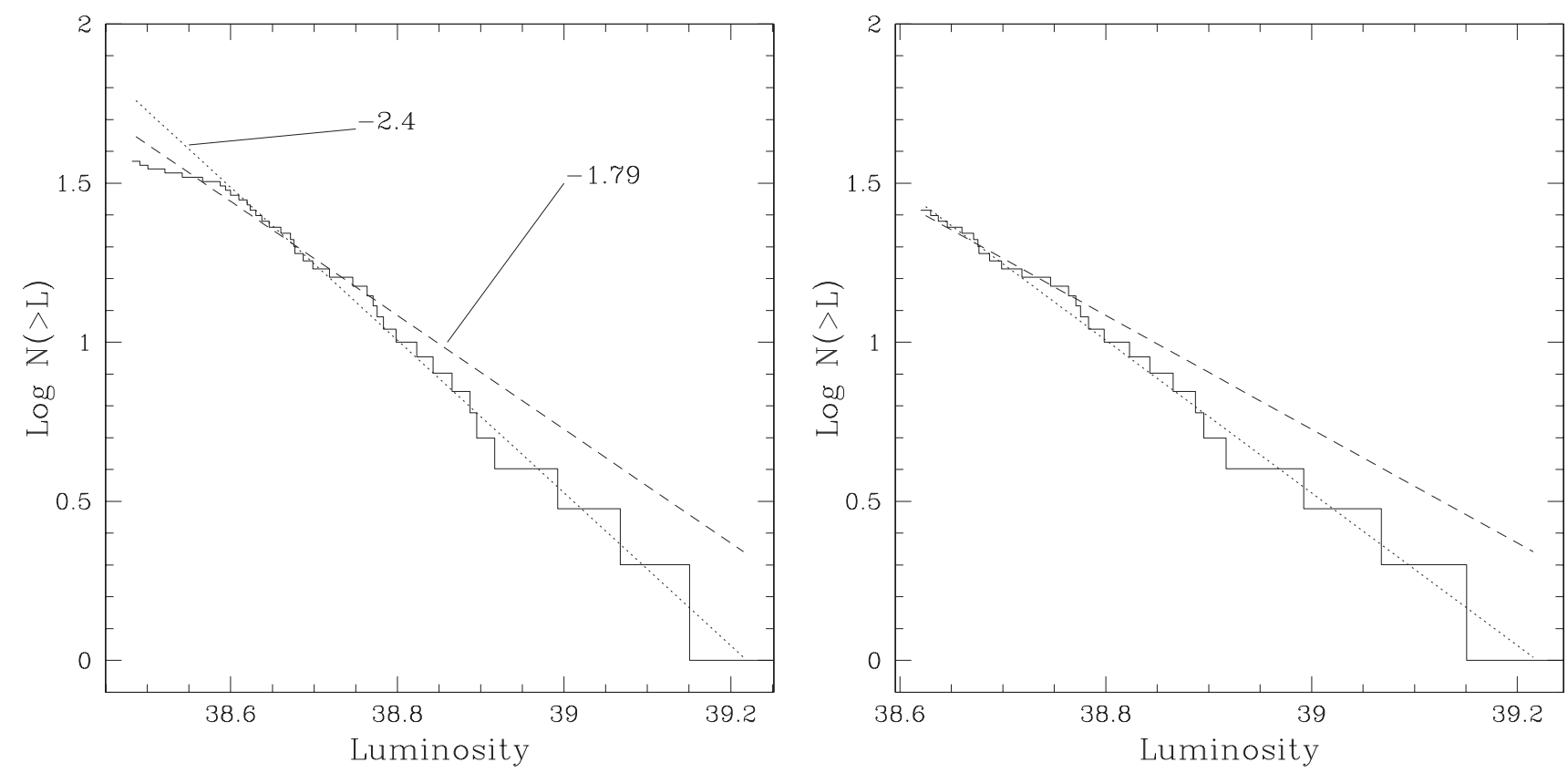

Fig. 7. The luminosity function of the sources in NGC $5846(0.3-10 \mathrm{keV}$ energy band). The dashed slope represents the value found by Shirey et al. (2001) for the M 31 sources, although at lower $L_{\mathrm{x}}$, normalized to the present data. The -2.4 value appears to better represent the NGC 5846 data than the flatter function. Left panel: all sources listed in Table 5. Right panel: only sources with a Signal-to-noise ratio greater than 3.4.

Table 2. Results of spectral fitting using the XSPEC package. Counts are obtained in different regions as described. A combination of a plasma code (MEKAL) and Power law (with $\Gamma$ fixed at a value of 1.84 , best fit from the $1^{\prime}-2^{\prime}$ region) models is used. Errors on kT (fifth column) represent the $90 \%$ probability intervals. d.o.f. (sixth column) stands for Degrees of Freedom.

\begin{tabular}{|c|c|c|c|c|c|}
\hline Region & $\begin{array}{l}\text { Counts } \\
\pm \text { error }\end{array}$ & $\begin{array}{r}\text { Energy } \\
(\mathrm{keV})\end{array}$ & $\begin{array}{c}N_{\mathrm{H}} \\
\left(10^{-20}\right)\end{array}$ & $\begin{array}{l}k T \\
\text { error }\end{array}$ & $\begin{array}{l}\chi_{\nu}^{2} \\
\text { (d.o.f.) }\end{array}$ \\
\hline $\begin{array}{l}\text { High surface } \\
\text { brightness } \\
\text { within } 30^{\prime \prime}\end{array}$ & $\begin{array}{l}1652 \\
\pm 41\end{array}$ & $0.3-1.9$ & 6.6 & $\begin{array}{l}0.62 \\
0.59-0.65\end{array}$ & $\begin{array}{l}0.8 \\
(36)\end{array}$ \\
\hline $\begin{array}{l}\text { Low surface } \\
\text { brightness } \\
\text { within } 30^{\prime \prime}\end{array}$ & $\begin{array}{l}1865 \\
\pm 43\end{array}$ & $0.3-2.3$ & 6.3 & $\begin{array}{l}0.59 \\
0.57-0.62\end{array}$ & $\begin{array}{l}1.2 \\
(62)\end{array}$ \\
\hline Ann $0.75-1^{\prime}$ & $\begin{array}{l}3474 \\
\pm 62\end{array}$ & $0.3-2.4$ & 12 & $\begin{array}{l}0.60 \\
0.58-0.61\end{array}$ & $\begin{array}{l}1.00 \\
(54)\end{array}$ \\
\hline Ann $1^{\prime}-2^{\prime}$ & $\begin{array}{l}10872 \\
\pm 11\end{array}$ & $0.4-2.6$ & 14 & $\begin{array}{l}0.67 \\
0.65-0.68\end{array}$ & $\begin{array}{l}1.4 \\
(98)\end{array}$ \\
\hline Ann $2^{\prime}-4^{\prime}$ & $\begin{array}{l}5788 \\
\pm 102\end{array}$ & $0.4-1.8$ & 15 & $\begin{array}{l}0.81 \\
0.79-0.84\end{array}$ & $\begin{array}{l}1.0 \\
(51)\end{array}$ \\
\hline
\end{tabular}

Note: There is no requirement of a Power law component in the $2^{\prime}-4^{\prime}$ region.

not covered by the CCD is also masked out. The net counts have then been binned to obtain $\mathrm{a} \geq 2.5 \sigma$ significance in each bin.

It is apparent from the comparison that all regions have significantly different overall spectral shapes, and different positions of the peak. We have tried to fit the data with current spectral models, as summarized in Table 2. We stress that the results presented here are neither final nor the best possible, but their purpose is to give a simplified parameterization of the average gas properties. We have used a combination of plasma line code and simple thermal or power law models. We find that (i) reasonable fits require 2 components out to $2^{\prime}$ radius. Either a power law or Bremsstrahlung are needed to account for a residual high energy tail and extreme low energy absorption that a fit with only a plasma code required for the low energy range would produce. (ii) There is no strong evidence that one model plasma code should be preferred. 


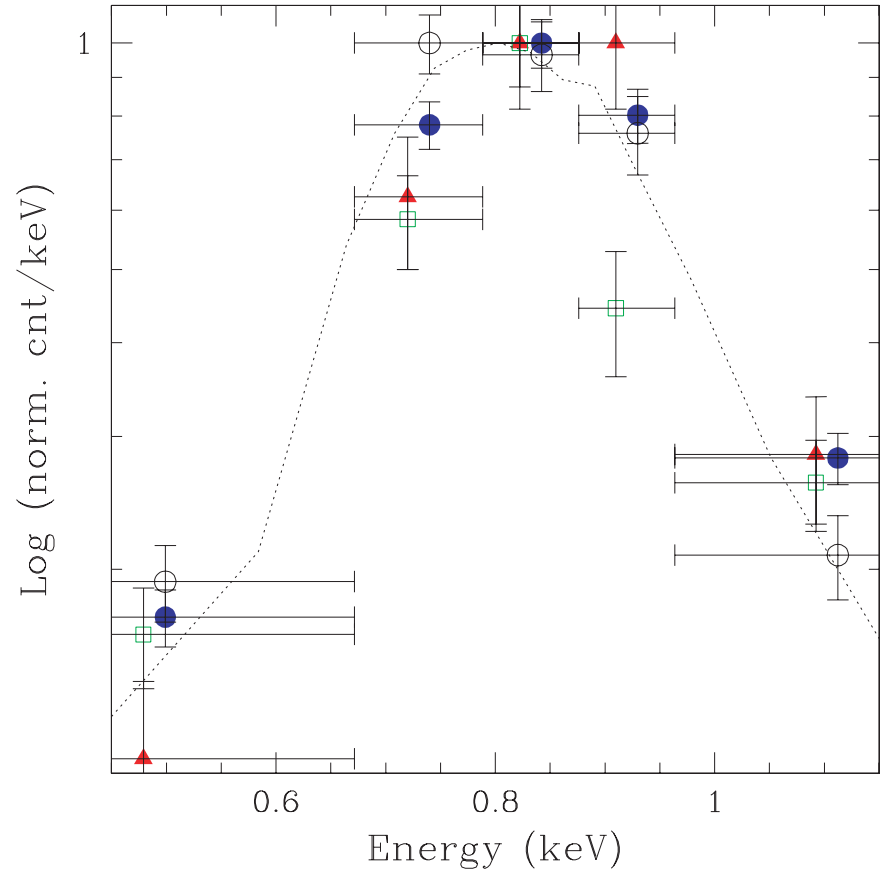

Fig. 8. The energy distribution of the photons in 4 regions within a radius of 0.75 at the galaxy center. All distributions are normalized to 1 at their peak, and are binned in the same energy bins for easy comparison. Filled circles and triangles are for the innermost regions, representative of the higher and lower surface brightness regions at the center, respectively. Open squares and open circles refer to regions at distances $\sim 0.5 \mathrm{~S}$ and $\mathrm{ENE}$ of the center, respectively. Horizontal error bars indicate the size of the energy bins. Points are plotted offset from the center of the bin to avoid confusion. The dotted line indicates the average energy distribution of the photons in the $0.5-0.75$ region. A finer energy binning is used for this region. Point sources detected at $2.5 \sigma$ and above have been masked out from all regions.

We used models based on both the Raymond-Smith code (hereafter Raymond code; Raymond \& Smith 1977) and the MEKAL code (e.g., Kaastra et al. 1996). The MEKAL code includes a better treatment of the Iron $\mathrm{L}$ lines near $1 \mathrm{keV}$ according to recent measurements and calculations of atomic parameters (Liedahl et al. 1995). The MEKAL code indeed yielded slightly better fits than the Raymond code (see, e.g., Fig. 10), but the required $N_{\mathrm{H}}$ is significantly higher than for the Raymond model, and than the line-of-sight value. (iii) The fits are not sensitive to the abundance assumed; (iv) we find significant residuals at $\sim 1.8 \mathrm{keV}$ and below $\sim 1 \mathrm{keV}$ (see Fig. 10 ), no matter what combination of models and parameters we use. We have used the latest calibration provided by the ASC/CXC, which significantly improve the quality of the fits over previous versions; however, we cannot exclude residual instrument calibration uncertainties.

Leaving both $k \mathrm{~T}$ and $\Gamma$ as free parameters, the best fit temperatures derived from the MEKAL model are not significantly different among the regions considered within $2^{\prime}$, in spite of the different photon distribution shown in Fig. 9. The power law slopes instead have very different
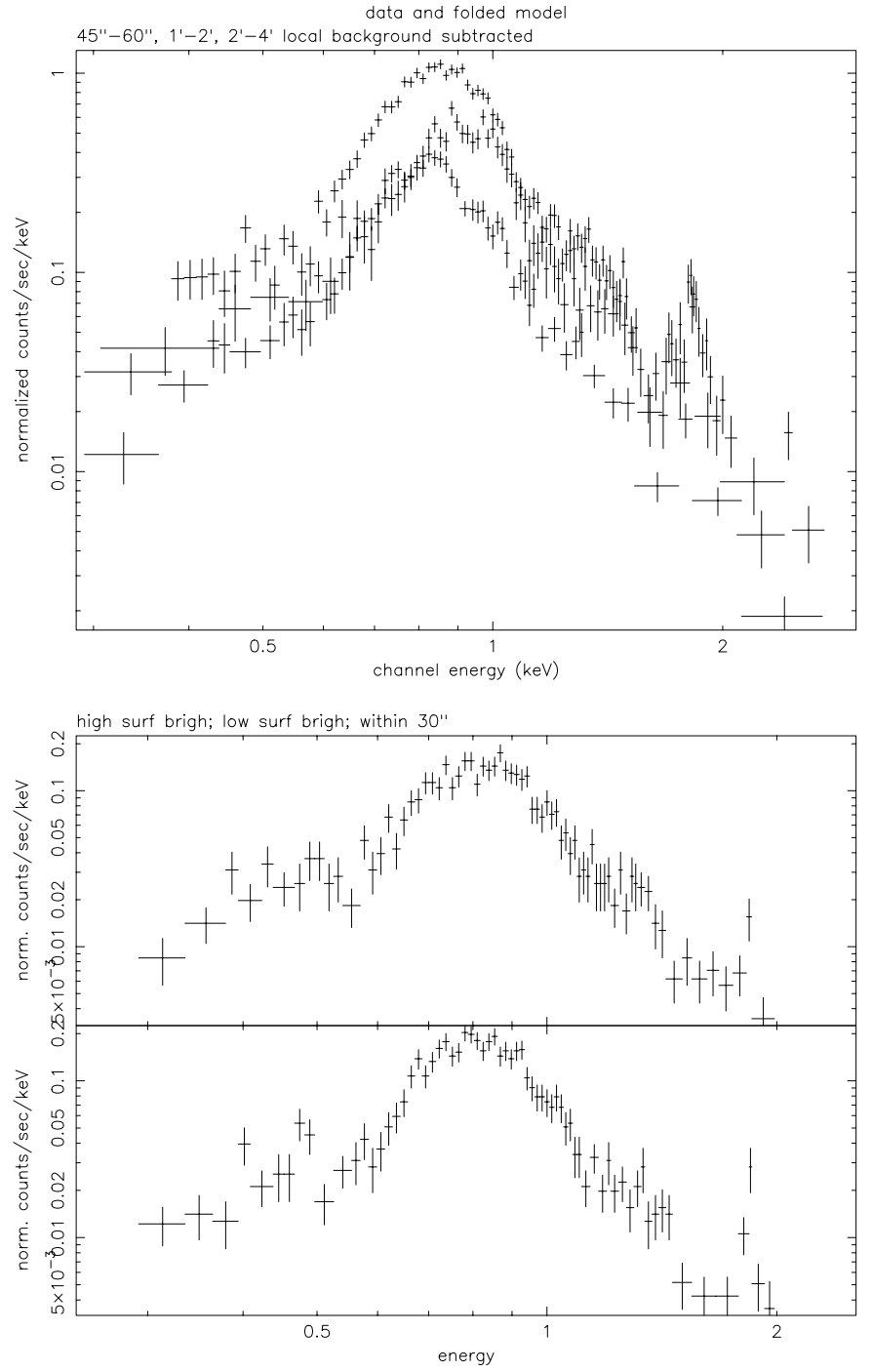

Fig. 9. The energy distribution of the photons in 3 adjacent annuli with "inner-outer" radii of $45^{\prime \prime}-60^{\prime \prime}, 1^{\prime}-2^{\prime}$ and $2^{\prime}-4^{\prime}$, and in 2 regions of higher and lower surface brightness within the inner $30^{\prime \prime}$. Detected point sources have been masked out. The background is obtained from the easternmost corner of the CCD field (see text) and subtracted in the outer annuli (top panel) normalized by the respective areas. The net counts are binned to retain a $2.5-3 \sigma$ significance in each bin.

best fit values ( $\Gamma$ in the range $0.15-2.5$ ), however with large errors. If we fix the power law slope to a common value (of $\Gamma=1.84$, best fit value from the $1^{\prime}-2^{\prime}$ region), we find that the temperature of the plasma increases towards the outside, from the low surface brightness central feature to the $2^{\prime}-4^{\prime}$ annulus. The low and high surface brightness features have formally different best fit temperatures, however the difference is not statistically significant (see Table 2). The $N_{\mathrm{H}}$ parameter is consistent with the line of sight value only in the very inner regions.

\section{Discussion}

The complexity of the emission in NGC 5846 is self-evident from the maps and from the comparison of the images at 


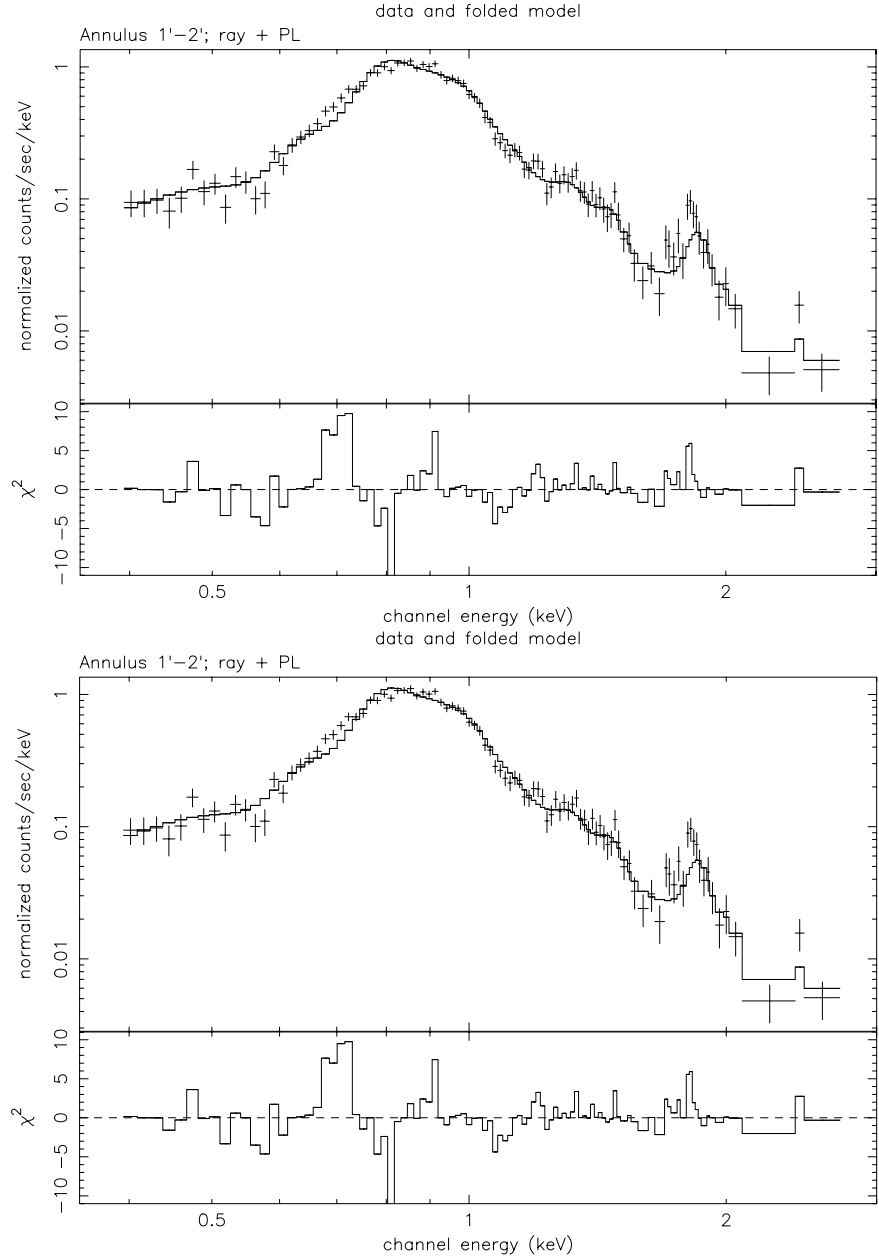

Fig. 10. Spectrum of the $1^{\prime}-2^{\prime}$ annulus, fitted with $1 T$ plasma code and a power law. The best fit parameters are: top panel: $N_{\mathrm{H}}=1.4 \times 10^{21} \mathrm{~cm}^{-2} ; k T($ MEKAL) $=0.66$; $\Gamma(\mathrm{PL})=1.8 ; \chi_{\nu}^{2}=1.4 ;$ bottom panel: $N_{\mathrm{H}}=7.5 \times 10^{20} \mathrm{~cm}^{-2}$; $k T($ Raymond $)=0.78 ; \Gamma(\mathrm{PL})=1.7 ; \chi_{\nu}^{2}=1.7$. Abundances are in both cases $1 \times$ solar. Data are fitted in the $0.4-2.6 \mathrm{keV}$ range. Local background is subtracted.

different energies. The high-energy band, above $\sim 3 \mathrm{keV}$, is completely dominated by a collection of individual sources that are now becoming a common feature in early type galaxies. In the soft energies, a very complex morphology is observed within the central $1^{\prime}-2^{\prime}$, with accompanying complexity in the gas physical and chemical properties, while a more regular (i.e., diffuse) gas distribution is observed at larger radii.

\subsection{Individual sources}

As already suggested by the crude properties of the low-luminosity early-type galaxies observed with Einstein (Fabbiano et al. 1992), and then measured with ASCA and BeppoSAX (Matsushida et al. 1994; Matsumoto et al. 1997; Pellegrini 1999; Trinchieri et al. 2000), a population of individual sources, most likely low mass $\mathrm{X}$ ray binaries, constitutes the dominant source of emission at high energies in "normal" early-type galaxies of all luminosities. A thorough study of this population is now possible with Chandra, due to its high spatial resolution and sensitivity. An example of such detailed study in the low-luminosity elliptical NGC 4697 is presented by Sarazin et al. $(2000,2001)$ where $\sim 90$ sources with luminosities above $\sim 5 \times 10^{37} \mathrm{erg} \mathrm{s}^{-1}$ are detected. A smaller though substantial population is also measured in NGC 1553 (Blanton et al. 2001), with $\sim 40$ sources above $\sim 3 \times 10^{38} \mathrm{erg} \mathrm{s}^{-1}$.

We detected $\sim 40$ sources in NGC 5846, all with luminosities above $\sim 3 \times 10^{38} \mathrm{erg} \mathrm{s}^{-1}$, which is higher than the Eddington luminosity for a $1 M_{\odot}$ accreting object. Ultra-Luminous Sources (ULS) have been known to exist in spiral galaxies for quite some time (Fabbiano 1989) but more examples of these non-nuclear bright sources are becoming available with Chandra data in both spiral (e.g. Tennant et al. 2001; Prestwich 2002) and elliptical galaxies (e.g. Blanton et al. 2001 and Sarazin et al. 2001). The brightest sources $\left(L_{\mathrm{X}}>2 \times 10^{39} \mathrm{erg} \mathrm{s}^{-1}\right)$ so far have almost esclusively been associated with spiral galaxies and regions of star formation activity (e.g., Zezas et al. 1999; Roberts \& Warwick 2000; Fabbiano et al. 2001), and are known to vary both in intensity and in spectral characteristics (Mizuno et al. 2001), supporting the idea that at least most of them are powered by a black hole. If the accretion is isotropic, these ULS would require intermediate-mass black holes, which are however inconsistent with measured inner disk temperatures (Kubota et al. 2001), and either anisotropic or beamed emission has been proposed to explain their high luminosities (King et al. 2001; Körding et al. 2002).

Although not as extreme, several point sources emitting above the Eddington luminosity are now also found in "normal" early-type galaxies, in locations without any association with star formation activity. A recent discussion on binary sources in early-type galaxies associates most of them, in particular the brighter ones, with the globular cluster population. In NGC 1399, Angelini et al. (2001) remark that the average luminosity of the globular cluster sources is higher than the rest of them. A high percentage of the sources in both NGC 4697 and NGC 1553 are also associated with globular clusters. As these three galaxies cover a large range in $L_{\mathrm{X}} / L_{B}$ ratio, it seems likely that a large fraction of $\mathrm{X}$-ray sources is associated with the globular cluster population in all early-type galaxies. It is quite possible that the same association exists for sources in NGC 5846, which has a rich population of them (Forbes et al. 1997 detect over 1200 globular clusters using WFPC2 images from HST). However, this is not at all what is observed in our Galaxy or M31, where no sources brighter than the Eddington limit are found associated with Globular clusters.

The comparison of the luminosity distributions of the sources in the three early-type galaxies available (data on NGC 1553 and NGC 4697 from the literature) indicates a lack of high-luminosity sources in NGC 5846 relative to the other two (i.e., a steeper slope). We cannot confirm a flattening in the luminosity function at low luminosities, 
which is most likely an effect of incompleteness. NGC 5846 is $\sim 50 \%$ more distant than NGC 1553 and $\sim 100 \%$ more than NGC 4697, the observing time on NGC 5846 was somewhat shorter than for the other two galaxies, and a much more significant component of hot gas is present in NGC 5846, therefore our limiting threshold is higher ( $\sim 6 \times$ than for NGC 4697, and only marginally than for NGC 1553), so we are not sampling the lower luminosity range as well as in these two galaxies. Furthermore, given the larger distance, our source confusion may be more severe. We expect this to be most relevant at the lower end of the luminosity distribution (brighter sources being rarer, and their luminosities being dominant over the addition of smaller contributions), which could artificially populate the low-to-intermediate luminosity range considered, resulting in a steeper relation. We therefore cannot claim that a break exists in the luminosity function of NGC 5846, since the flattening observed below $L_{\mathrm{X}} \sim 4 \times 10^{38} \mathrm{erg} \mathrm{s}^{-1}$ is almost surely due to incompleteness at the low end of the $L_{\mathrm{X}}$ distribution. However, we detect a steeper slope above $\sim 6 \times 10^{38} \mathrm{erg} \mathrm{s}^{-1}$ relative to the luminosity functions of NGC 1553 and NGC 4697.

\subsection{Large scale distribution of the hot gas}

Given the small field of view of the S3 detector relative to the full spatial extent of the X-ray emission in this object, little can be said about the large-scale structure of the gas. In particular, the $\mathrm{W}$ quadrant is only covered out to $r \sim 2^{\prime}-3^{\prime}$, before reaching the edges of the CCD. To the $\mathrm{E}$, the coverage is better, but only out to $r \sim 4^{\prime}-5^{\prime}$ for a large fraction of the galaxy.

As shown by the radial profiles in Figs. 3 and 4, extended emission is detected out to $\gtrsim 6^{\prime}$, i.e., to the boundary of the CCD field of view. The shape of the photon distribution appears broader above than below $\sim 1 \mathrm{keV}$, and shows azimuthal asymmetries at all energies. In particular, a strong enhancement is observed in the $0^{\circ}<\mathrm{PA}<120^{\circ}$ region, between $\sim 1^{\prime}-2.5$ radius, more pronounced below $1 \mathrm{keV}$. Moreover, the $0^{\circ}<\mathrm{PA}<90^{\circ}$ sector profile appears flatter than the remaining sectors, mostly below $\sim 1 \mathrm{keV}$. The significant azimuthal asymmetries in the photon distribution prevents us from parameterizing the radial profile of the emission with a "King-type" profile as done in the past. While this kind of profile might still be valid at larger radii, the inner region clearly requires a much more complex model.

The spectral data also indicate that a complex spectral model is needed, which appears to vary even within relatively small regions. At large radii $\left(r>2^{\prime}\right)$ only the eastern portion of the galaxy is covered, and the spectral photon distribution can be fitted reasonably well with a single-temperature plasma with $k T \sim$ of $0.8 \mathrm{keV}$. It is likely that at these radii the turmoil affecting the innermost parts of the galaxy is less severe and the gas is in an energetically stabler configuration. However, the lack of coverage at larger radii prevents us from a more complete analysis of these regions.

\subsection{Small scale properties of the hot gas}

The central region of NGC 5846 shows complex morphology in the form of a prominent "hook" at the center, plus several "blobs" and filaments to the NNE at $\sim 1^{\prime}-3^{\prime}$ radius and towards $\mathrm{S}$, that had already been seen in the ROSAT HRI images (Trinchieri et al. 1997a). The spectral properties are not the same in different regions, suggesting a rather complex physical state of the interstellar medium.

Complex gas morphologies are now more common as Chandra data on bright early-type galaxies become available. NGC 1553 (Blanton et al. 2001) and NGC 4636 (Jones et al. 2001) show a spiral-like feature at the center, NGC 4696 (Sanders \& Fabian 2001) exhibits a spiral plume-like feature, and a disturbed inner halo is seen in NGC 1399 (Angelini et al. 2001), to name a few. In several objects, the peculiar morphologies are associated with central activity, in the form of an extended radio source (e.g., NGC 1399) or of a previously unknown (obscured, or low-luminosity) X-ray central source (e.g. NGC 1553), but not in all (e.g., NGC 4636 has neither an X-ray nucleus nor a strong radio source). However, what makes NGC 5846 galaxy unique (up to now!) relative to the other examples is the close agreement found between the morphology in the X-ray gas and in the optical line-emitting gas $(\mathrm{H} \alpha+[\mathrm{N} \mathrm{II}])$ and dust. Several objects show complex lineemitting morphologies, but so far claims of similarities do not extend to the very inner regions, like in NGC 5846 (see Fig. 11). Even the morphological similarity found for NGC 1553 with the ROSAT HRI data (Trinchieri et al. 1997a) does not appear to extend to smaller scales (Blanton et al. 2001).

Morphological agreement on arcmin scales was already suggested by the ROSAT HRI data (Trinchieri et al. 1997a; Goudfrooij \& Trinchieri 1998). With the present data, the morphological similarity with the optical line emission is brought an order of magnitude closer in, to scales as small as a few arcsec (cf. Fig. 11), equivalent to a few hundred parsecs, while no equivalent distortions are seen in the red continuum image (Fig. 2). Dust absorption, also detected in optical images of NGC 5846 (Goudfrooij \& Trinchieri 1998; Forbes et al. 1997), also displays a very similar filamentary structure extending down towards the nucleus.

The close similarity in the distributions of the X-ray and $\mathrm{H} \alpha+[\mathrm{NII}]$ emission is rather stunning. In some aspects it is almost an exact replica in both morphology and intensity variations, in others at least the morphology is similar. For example, the "two-horned" $\mathrm{H} \alpha$ structure is reflected in a stronger and longer "horn" in X-rays in the innermost crosscut (right "horn"), while the left feature is visible as a distortion in the gas at larger radii. Horizontal and vertical cuts at different locations through the X-ray and $\mathrm{H} \alpha+[\mathrm{NII}]$ images confirm the visual 

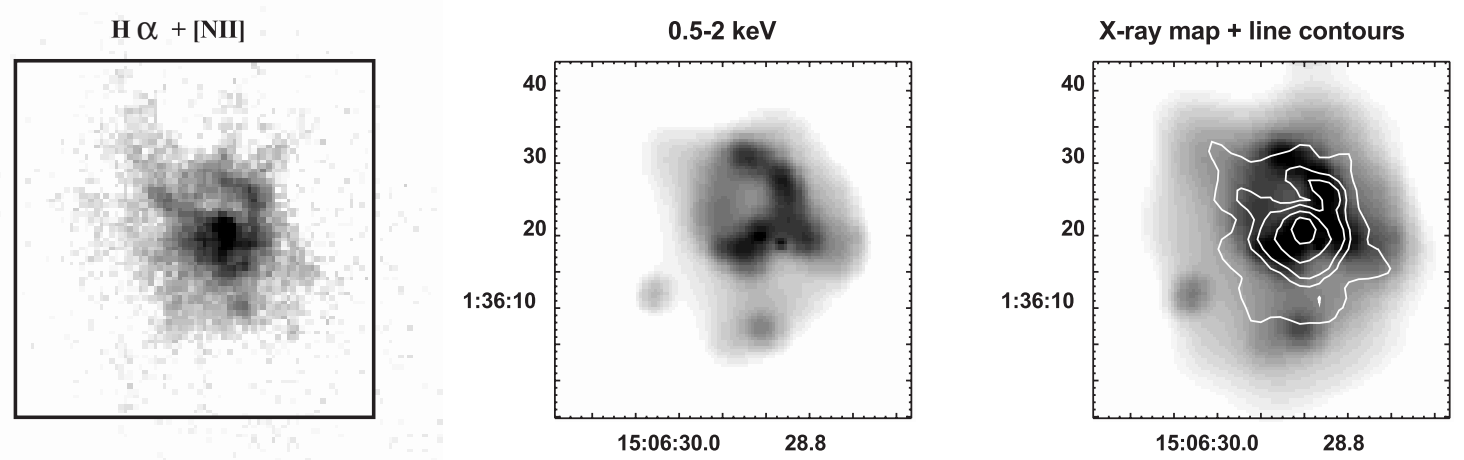

Fig. 11. Comparison of the innermost region of NGC 5846 in the $\mathrm{H} \alpha+[\mathrm{N} I \mathrm{I}]$ lines and in $\mathrm{X}$ rays $(0.5-2.0 \mathrm{keV})$. The data are displayed on the same scale. $\mathrm{H} \alpha+[\mathrm{N}$ II] contours on the X-ray smoothed image are shown in the right panel. $\mathrm{H} \alpha+[\mathrm{N}$ II] line filter images were obtained with EFOSC1 at the ESO 3.6-m telescope (see Trinchieri \& di Serego Alighieri 1991).

impression of similar pattern at different intensities (Fig. 12) and give a more quantitative view of the large intensity contrasts present at both wavelengths. In crosscut 2, X-ray and $\mathrm{H} \alpha+[\mathrm{N}$ II] emission are similar both in extent $\left(\leq 30^{\prime \prime}\right)$ and in relative intensities $(\sim \times 2$ from peak to shoulder), and a similarly broad base is present in crosscuts 3 and 4, although the very intense $\mathrm{H} \alpha$ peak has no correspondence in the X-rays. The comparison of crosscuts 1 and 6 would suggest that the panels have been exchanged, since the X-ray cut of region 1 closely resembles the $\mathrm{H} \alpha$ cut of region 6 and vice-versa. Finally, crosscut 5 appears somewhat more extended in X-rays, and with a larger plateau.

What is the nature of these intermediate- and smallscale morphological structures? The galaxy is at the center of a small group, and the large-scale gas distribution shown by the PSPC data is regular and almost azimuthally symmetric (Finoguenov et al. 1999). The galaxy is not associated with a strong, extended radio source: A partially-resolved radio source is detected at 1.4 and $8.4 \mathrm{GHz}$ with the VLA (Filho et al. 2000; Möllenhoff et al. 1992), at a flux density that is several orders of magnitude fainter than cores of more classical radio galaxies (Fanaroff \& Riley 1974) and closer in power to the weak cores of Seyfert galaxies. We therefore do not expect it to cause a significant displacement of the hot gas as observed in stronger, extended radio sources. We also do not find any evidence for the presence of an X-ray-bright nuclear source which might cause turmoil in the center.

A "high-speed encounter" has been postulated between NGC 5846 and its close companion NGC 5850 (Higdon et al. 1998), mainly to explain the latter galaxy's peculiar morphology. This is not likely to explain the disturbed appearance in the hot gas distribution of NGC 5846, since the effects should be visible also at larger distances and in particular towards the spiral galaxy itself. If anything, the X-ray contours towards NGC 5850 (i.e., to the E) appear steeper on scales $>5^{\prime}$, which might argue for gas compression if the galaxy is approaching (cf. Fig. 1 in Finoguenov et al. 1999; note that Higdon et al. 1998 discard a ram-pressure stripping explanation for the morphology of NGC 5850, due to the lack of extended Xray emission in the system). At smaller scales, the distortion is towards the NNE, and its shape is more reminiscent of a tidal effect of an escaping object or a captured object spiraling in towards the galaxy centre. The existence of a similar spur in the morphology of the optical emissionline gas also suggest influence from an "external source". The kinematics of the $\mathrm{H} \alpha+[\mathrm{N}$ II $]$ emission is found to be irregular, with steep velocity gradients in the the N-S direction, and a sudden change of sign of velocity towards the S. Conversely, the kinematics of the stellar component is regular, showing slow rotation (Caon et al. 2000). The kinematically distinct inner gas component supports the idea of external accretion, when the gas has not yet settled into an equilibrium configuration. A similar interpretation was put forward by Sparks et al. (1989) and de Jong et al. (1990) for the nature of the optical nebulosity in NGC 4696, which they associate with a small, gas-rich galaxy that has fallen in and has been stripped of its gas and dust.

Given the likely external origin of the optical nebulosity and dust in NGC 5846, the idea of a causal connection between the dust and optical nebulosity on one side and the hot gas on the other may seem counterintuitive, since the hot gas most likely has an "internal" origin, coming from mass loss of old stars (e.g., Fabbiano 1989). Sparks et al. (1989) and de Jong et al. (1990) independently suggested that the enhancement of the X-ray emission associated with the colder gas and dust is caused by electron conduction. The hot electrons thus act as cooling agent for the hot gas, while causing transient heating for dust grains and plausibly even acting as ionizing source for the optical emission-line gas (see also Voit \& Donahue 1997; Goudfrooij \& Trinchieri 1998). This model was suggested to explain the larger-scale similarities in NGC 5846 observed with the ROSAT HRI (Trinchieri et al. 1997a; Goudfrooij \& Trinchieri 1998). We can now give a quantitative estimate of the expected luminosities in the features observed at small scales.

We have estimated an average X-ray surface brightness in the "hook" of $\sim 7$ counts $\operatorname{arcmin}^{-2}$, corresponding to 
region $1 \mathrm{X}$-ray

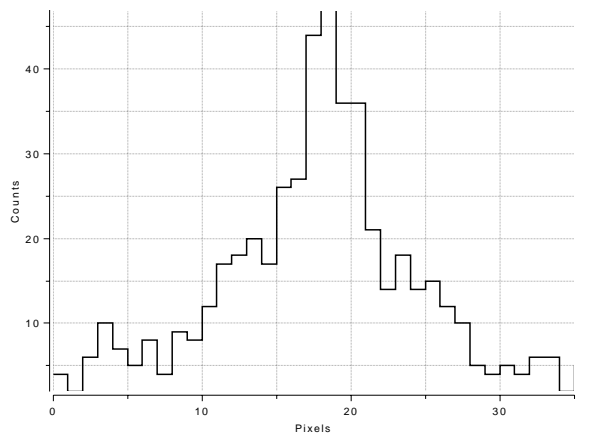

region $1 \mathrm{H} \alpha+[\mathrm{N} \mathrm{II}]$

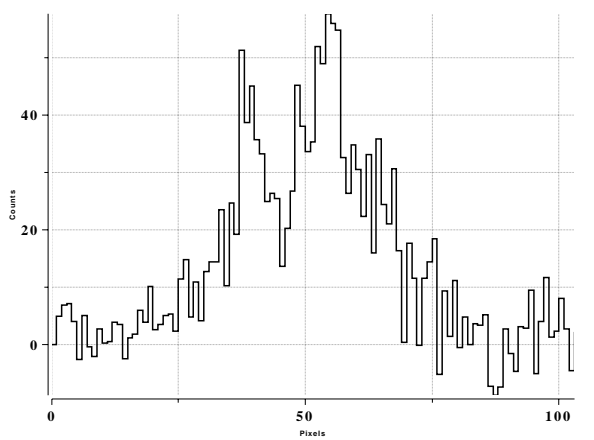

region $4 \mathrm{X}$-ray

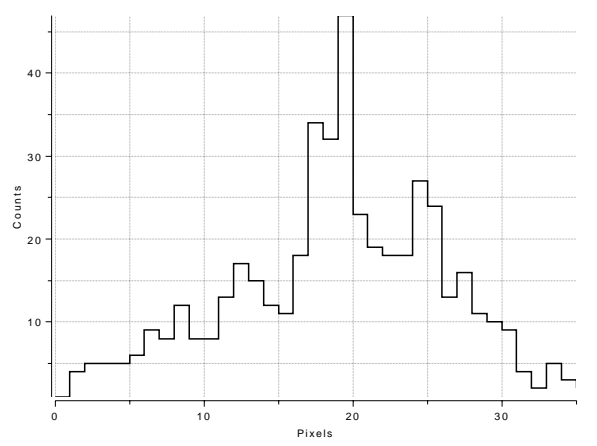

region $4 \mathrm{H} \alpha+[\mathrm{N}$ II]

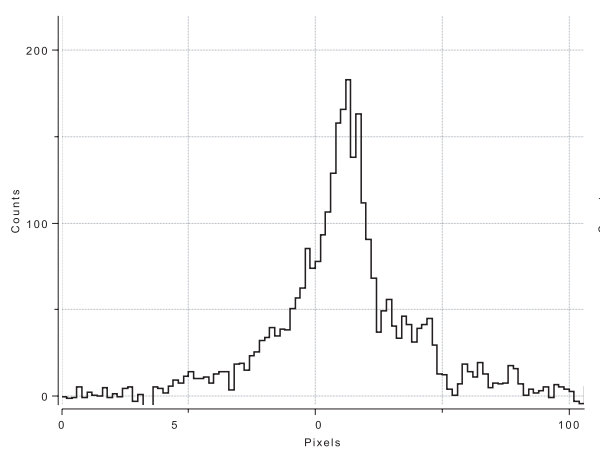

region $2 \mathrm{X}$-ray

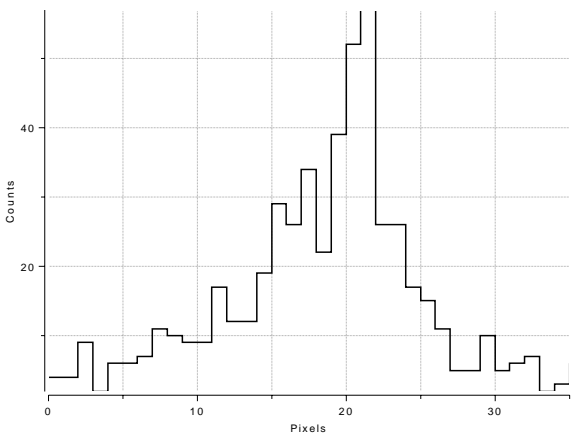

region $2 \mathrm{H} \alpha+[\mathrm{N}$ II $]$

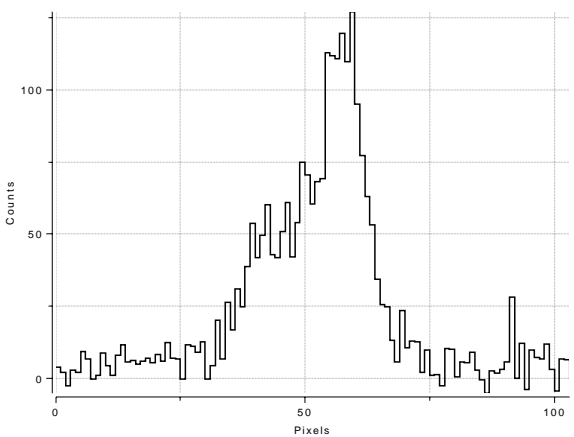

region $5 \mathrm{X}$-ray

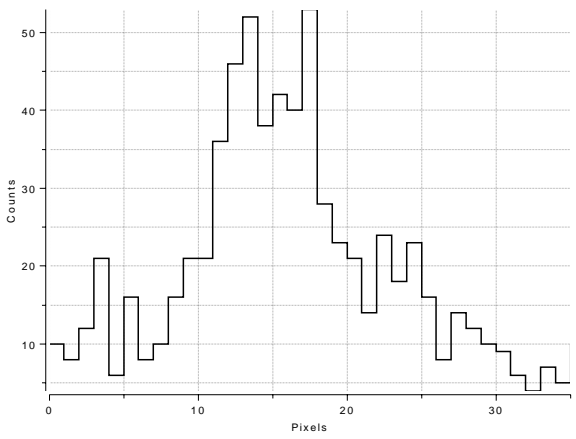

region $5 \mathrm{H} \alpha+[\mathrm{N}$ II $]$

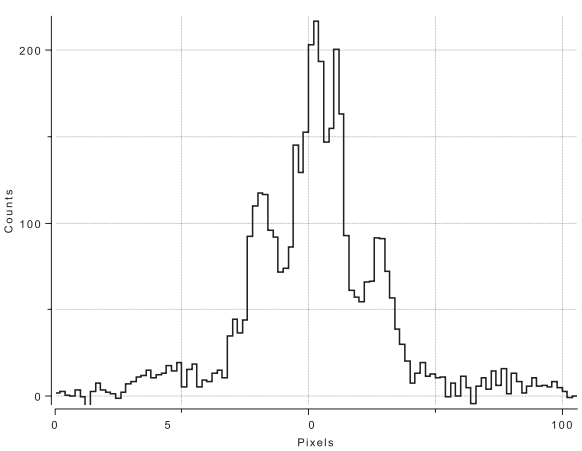

region $3 \mathrm{X}$-ray

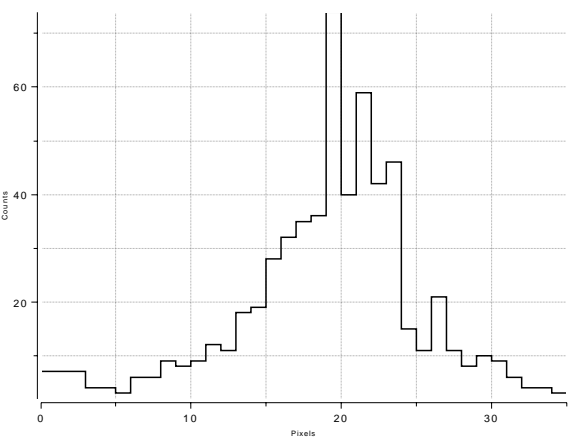

region $3 \mathrm{H} \alpha+[\mathrm{N} \mathrm{II}]$

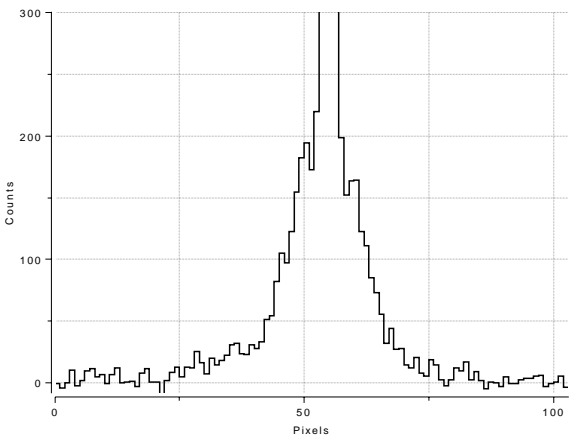

region $6 \mathrm{X}$-ray

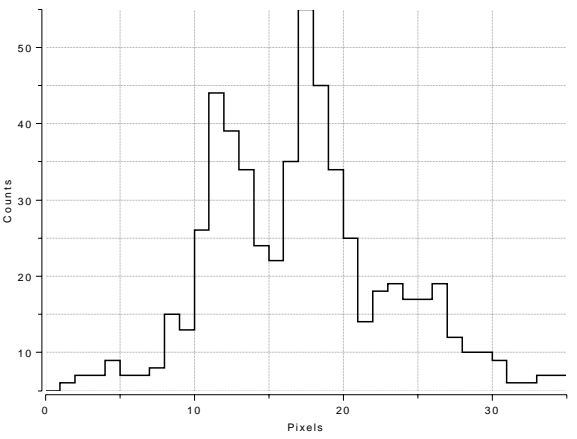

region $6 \mathrm{H} \alpha+[\mathrm{N} \mathrm{II}]$

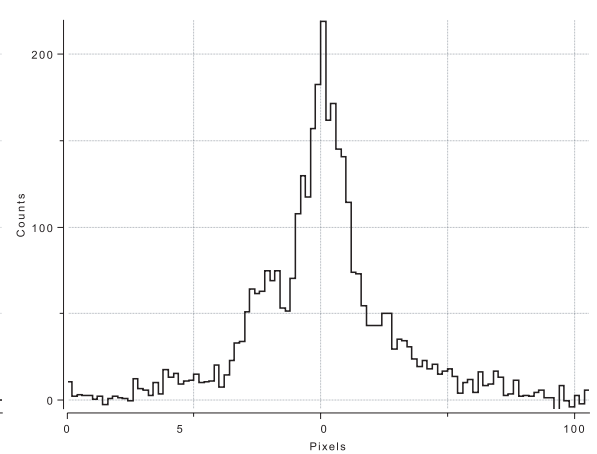

Fig. 12. Cuts along the horizontal (1 through 4) and vertical (5 and 6) axes through the central regions of NGC 5846 in the X-ray (first and third row) and the $\mathrm{H} \alpha+\left[\mathrm{N}\right.$ II] (second and fourth row) images. Horizontal scale is $70^{\prime \prime}$, and the slits are positioned in the same place in the two images. Pixel sizes are $2^{\prime \prime}$ in the X-ray and 0.'675 in the H $\alpha+[\mathrm{N}$ II] image. Cuts are across the high and low surface brightness central features as shown in Fig. 13. The sharp peak in the H $\alpha$ cut \# 3 has been set off-scale to better show the lower part of the curve. Vertical scales could not be set equal in all panels, due to the different dynamical ranges in different regions. 


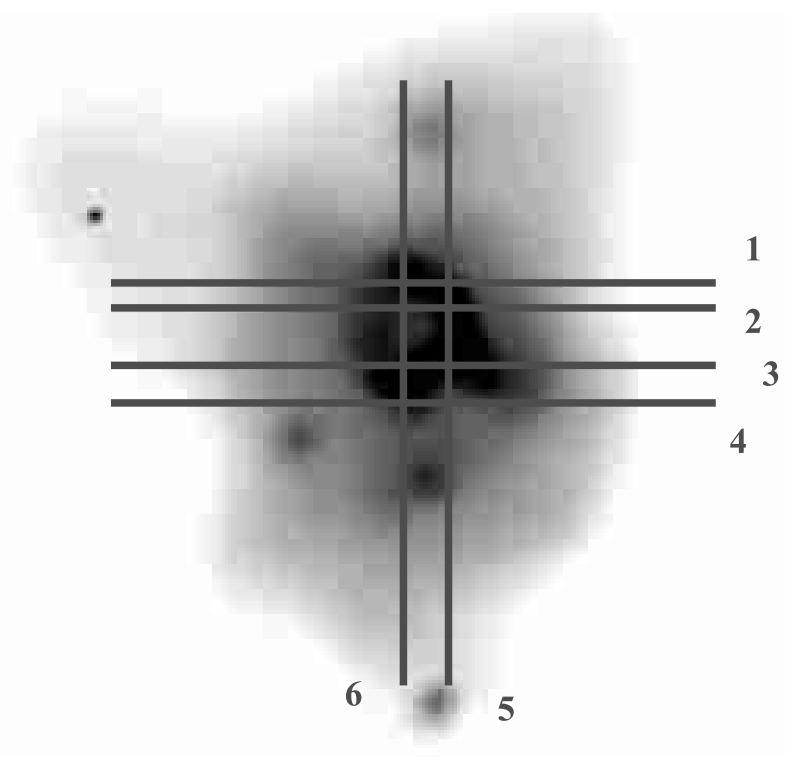

Fig. 13. Position of the cuts used to derive the plots in Fig. 12.

$\sim 1.4 \times 10^{38} \mathrm{erg} \mathrm{s}^{-1} \mathrm{pc}^{-2}$ at the adopted distance. Although we do not have a very precise assessment of the temperature of the gas, the derived density depends only weakly on temperature. Hence we choose a uniform temperature of $k T=0.6 \mathrm{keV}$. The choice of the assumed volume is however more critical, and we do not have direct information about the depth of the structure. Assuming that the emission comes from a narrow, bent ellipsoid, with depth equivalent to the narrow side, the average density of the hot gas is $n_{\mathrm{e}}=0.35 \mathrm{~cm}^{-3}$. Following Macchetto et al. (1996, as derived from Cowie \& McKee 1977), the predicted line flux is $L_{\mathrm{H} \alpha} \sim 2.4 \times 10^{40} \mathrm{erg} \mathrm{s}^{-1}$, assuming $1 \%$ of the available energy is radiated in the $\mathrm{H} \alpha+[\mathrm{N} \mathrm{II}]$ lines. This is to be compared to $L_{\mathrm{H} \alpha} \sim 1.3 \times 10^{40} \mathrm{erg} \mathrm{s}^{-1}$ observed. The good agreement between the two estimates reinforces the likelihood of the "heat conduction" model.

\section{Conclusions}

High resolution images of NGC 5846 obtained with Chandra show a disturbed hot gas morphology on arcsecond scale $\left(1^{\prime \prime}=140 \mathrm{pc}\right)$ with unprecedented details. The morphological peculiarities are coupled with a complex energy distribution of the photons. Using these highresolution observations, the previously reported similarity of the X-ray emission and the $\mathrm{H} \alpha+[\mathrm{N}$ II] features are now extended into the very inner regions of the galaxy, reinforcing the idea that there must be a close link between these two phases of the ISM. A viable interpretation of this phenomenon is that the gas ionization responsible for the optical line emission is provided by the electrons that also take care of the heat conduction within the hot gas.

About 40 point-like sources are found in NGC 5846, with $L_{\mathrm{X}}>3 \times 10^{38} \mathrm{erg} \mathrm{s}^{-1}$. The luminosity function of these sources appears steeper than any previously reported for early-type galaxies. However, we cannot exclude a bias at the low-luminosity end that might artificially increase the contribution of low-luminosity sources. We also cannot confirm the change in slope observed at low luminosities in other early-type galaxies.

Acknowledgements. We thank the whole Chandra team for help and support during the data analysis and for many useful discussions on the different aspects of the new data. In particular we thank E. Mandel and B. Joye for their continuous support and good will to improve the funtools/DS9 software, that we have heavily used in our analysis. This work has received partial financial support from the Italian Space Agency. PG was affiliated with the Astrophysics Division of the Space Science Department of the European Space Agency during part of this project.

\section{References}

Angelini, L., Loewenstein, M., \& Mushotzky, R. F. 2001, ApJ, 557, L35

Baum, S. A. 1992, PASP, 104, 848

Biermann, P. L., Kronberg, P. P., \& Schmutzler, T. 1989, A\&A, 208, 22

Blanton, E. L., Sarazin, C. L., \& Irwin, J. A. 2001, ApJ, 552, 106

Buson, L., Sadler, E. M, Zeilinger, W. W., et al. 1993, A\&A, 280, 409

Canizares, C. R. C., Fabbiano, G., \& Trinchieri, G. 1987, ApJ, 312,503

Caon, N., Macchetto, D., \& Pastoriza, M. 2000, ApJS, 127, 39

Cowie, L. L., \& Mckee, C. F. 1977, ApJ, 211, 135

de Jong, T., Nørgaard-Nielsen, H. U., Hansen, L., \& Jørgensen, H. E. 1990, A\&A, 232, 317

Fabbiano, G., Zezas, A., \& Murray, S. S. 2001, ApJ, 5541035

Fabbiano, G., Kim, D-W., \& Trinchieri, G. 1992, ApJS, 80, 645

Fabbiano, G. 1989, ARA\&A, 27, 87

Fanaroff, B. L., \& Riley, J. M. 1974, MNRAS, 167, 31

Filho, M. E., Barthel, P. D., \& Ho, L. C. 2000, ApJS, 129, 93

Finoguenov, A., Jones, C., Forman, W., \& David, L. 1999, ApJ, 514,844

Forbes, D. A., Brodie, J. P., \& Huchra, J. 1997, AJ, 113, 887

Forman, W., Schwarz, J., Jones, C., Liller, W., \& Fabian, A. C. 1979, ApJ, 234, L27

Forman, W., Jones, C., \& Tucker, W. 1985, ApJ, 293, 102

Goudfrooij, P., Hansel, L., Jørgensen, H.-E., \& Nørgaard-Nielsen, H. U. 1994, A\&AS, 105, 341

Goudfrooij, P., \& Trinchieri, G. 1998, A\&A, 330, 123

Higdon, J. L., Buta, R. J., \& Purcell, G. B. 1998, AJ, 115, 80

Hasinger, G., Burg, R., Giacconi, R., et al. 1998, A\&A, 329, 482

Hicks, A., Wise, M. W., Houck, J., \& Canizares, C. R. 2002, ApJ, submitted

Jones, C., Forman, W., Vikhlinin, A., et al. 2001, ApJL, submitted [astro-ph/0108114]

Kaastra, J. S., Mewe, R., Liedahl, D. A., et al. 1996, A\&A, 314,547

Kim, D.-W. 1989, ApJ, 346, 653

Kim, D.-W., \& Fabbiano, G. 1995, ApJ, 441, 182

King, A. R., Davies, M. B., Ward, M. J., Fabbiano, G., \& Elvis, M. 2001, ApJ, 552, L109

Körding, E., Falcke, H., \& Markoff, S. 2002, A\&A, 382, L13

Kubota, A., Mizuno, T., Makishima, K., et al. 2001, ApJ, 547, L119 
Leidahl, D. A., Osterheld, A. L., \& Goldstein, W. H. 1995, ApJ, 438, L115

Matsumoto, H., Koyama, K., Awaki, H., et al. 1997, ApJ, 482 133

Matsushita, K., Makishima, K., Awaki, H., et al. 1994, ApJ, 436, L41

Macchetto, F., Pastoriza, M., Caon, N., et al. 1996, A\&AS, 120,463

Mizuno, T., Kubota, A., \& Makishima, K. 2001, ApJ, 554, 128

Möllenhoff, C., Hummel. E., \& Bender, R. 1992, A\&A, 255, 35

Pellegrini, S. 1999, A\&A, 343, 23

Prestwich, A. H. 2002, in The High-Energy Universe at Sharp Focus: A Symposium of Chandra Science (ASP, San Francisco), in press [astro-ph/0108523]

Raymond, J. C., \& Smith, B. W. 1977, ApJS, 35, 419

Roberts, T. P., \& Warwick, R. S. 2000, MNRAS, 315, 98

Sanders, J. S., \& Fabian, A. C. 2001 [astro-ph/0109336]

Sarazin, C. L., Irwin, J. A., \& Bregman, J. N. 2000, ApJ, 544, L101

Sarazin, C. L., Irwin, J. A., \& Bregman, J. N. 2001, ApJ, 556, 533

Shields, J. C. 1991, AJ, 102, 1314

Shirey, R., Soria, R., Borozdin, K., et al. 2001, A\&A, 365, 195

Singh, K. P. Bhat, P. N., Prabhu, T. P., \& Kembhavi, A. K. 1995, A\&A, 302, 658
Sparks, W. B., Jedrzejewski, R. I., \& Macchetto, F. 1994, in The soft X-ray cosmos, ed. E. M. Schlegel, \& R. Petre, AIP Conf. Proc., 313, New York, 389

Sparks, W. B., Macchetto, F., \& Golombek, D. 1989, ApJ, 345, 153

Tennant, A. F., Wu, K., Ghosh, K. K., Kolodziejczak, J. J., \& Swartz, D. A. 2001, ApJ, 549, 43

Trinchieri, G., \& di Serego Alighieri, S. 1991, AJ, 101, 1647

Trinchieri, G., Kim, D.-W., Fabbiano, G., \& canizares, S. R. C. 1994, ApJ, 428, 555

Trinchieri, G., \& Noris, L. 1995, in Röntgenstrahlung from the Universe, ed. H.-U. Zimmermann, J. Trümper, \& H. Yorke, 397

Trinchieri, G., Noris, L., \& diSerego Alighieri, S. 1997a, A\&A, 326,565

Trinchieri, G., Fabbiano, G., \& Kim, D.-W. 1997b, A\&A, 318, 361

Trinchieri, G., Pellegrini, S., Wolter, A., Fabbiano, G., \& Fiore, F. 2000, A\&A, 364, 53

Voit, G. M., \& Donahue, M. 1997, ApJ, 486, 242

Zezas, A. L., Georgantopoulos, I., \& Ward, M. J. 1999, MNRAS, 308, 302 